\title{
On the influence of two-dimensional hump roughness on laminar-turbulent transition
}

Cite as: Phys. Fluids 32, 034102 (2020); https://doi.org/10.1063/1.5131577

Submitted: 14 October 2019 . Accepted: 07 February 2020 . Published Online: 02 March 2020

Juan Alberto Franco Sumariva (D), Stefan Hein, and Eusebio Valero
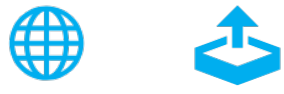

\section{ARTICLES YOU MAY BE INTERESTED IN}

Surface wave mechanism for directional motion of droplet on an obliquely vibrated substrate

Physics of Fluids 32, 031701 (2020); https://doi.org/10.1063/1.5143874

A comparative study on the large-scale-resolving capability of wall-modeled large-eddy simulation

Physics of Fluids 32, 035102 (2020); https://doi.org/10.1063/1.5144669

Nonlinear evolution of perturbations in high Mach number wall-bounded flow: Pressuredilatation effects

Physics of Fluids 32, 036101 (2020); https://doi.org/10.1063/1.5144420

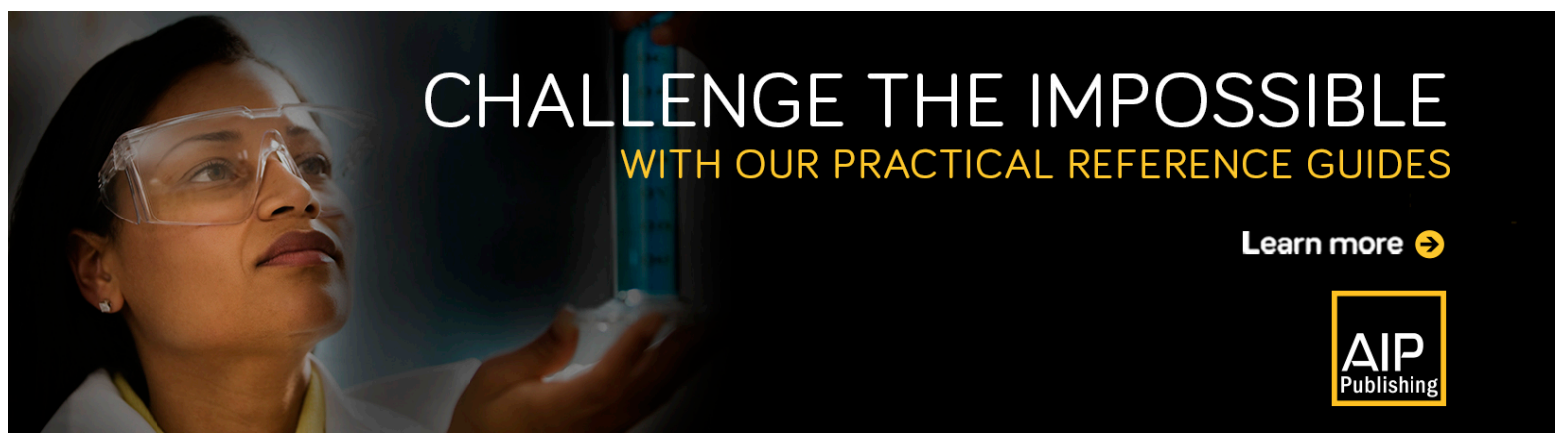




\title{
On the influence of two-dimensional hump roughness on laminar-turbulent transition
}

\author{
Cite as: Phys. Fluids 32, 034102 (2020); doi: 10.1063/1.5131577 \\ Submitted: 14 October 2019 - Accepted: 7 February 2020 • \\ Published Online: 2 March 2020
}

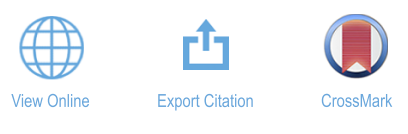

Juan Alberto Franco Sumariva, ${ }^{1,2, a)}$ (D) Stefan Hein, ${ }^{1}$ and Eusebio Valero ${ }^{2}$

\begin{abstract}
AFFILIATIONS
${ }^{1}$ Institute of Aerodynamics and Flow Technology, German Aerospace Center (DLR), Bunsenstrasse 10, 37073 Göttingen, Germany ${ }^{2}$ Universidad Politécnica de Madrid, Plaza Cardenal Cisneros 3, E-28040 Madrid, Spain
\end{abstract}

a) Author to whom correspondence should be addressed: Juan.Franco@dlr.de

\begin{abstract}
The presence of large surface irregularities such as humps, where the height is similar to the local boundary-layer (BL) displacement thickness, introduces regions of localized strong streamwise gradients in the base flow quantities. These large gradients can significantly modify the spatial development of incoming disturbances that lead to laminar-turbulent transition in wall-bounded flows [e.g., Tollmien-Schlichting (TS) waves]. Techniques such as Parabolized Stability Equations (PSE) are not suited for BL instability analysis in such regions: their formulation assumes that streamwise variations of base flow and disturbance quantities are small, allowing a marching procedure for their resolution. On the other hand, the Adaptive Harmonic Linearized Navier-Stokes (AHLNS) equations can handle these large streamwise gradients by using a fully elliptic system of equations, similar to Linearized Navier-Stokes (LNS), Harmonic LNS (HLNS), or Direct Numerical Simulation (DNS). Moreover, in AHLNS (as in PSE), a wave-like character of the instabilities is assumed, leading to a significant reduction in the number of streamwise grid points required compared with LNS, HLNS, or DNS computations. In the present study, an efficient combination of PSE and AHLNS is used to investigate the effect of height, length, and shape of a single hump placed on a flat plate in a two-dimensional flow field at $M a_{\infty}=0.5$ without pressure gradient. The effect of this hump on the spatial evolution of TS waves, in terms of $N$-factors, is presented. An expected laminar-turbulent transition onset, via the $e^{N}$ methodology, is also described. It is shown that the shape of the surface irregularity, together with the height and length, plays an important role for the location of laminar-turbulent transition onset in convectively unstable flows.
\end{abstract}

Published under license by AIP Publishing. https://doi.org/10.1063/1.5131577

\section{INTRODUCTION}

Laminar wings are one of the most promising research areas for reducing the overall drag of future aircraft. The design of such wings tries to extend the laminar properties of the incoming flow along the wing surface and therefore to delay the onset of laminarturbulent transition as much as possible. In such circumstances, the presence of surface irregularities such as steps, gaps, humps, and waviness becomes a critical aspect in the design process. In some cases, the presence of such irregularities may become unavoidable and, therefore, it is necessary to establish tolerance criteria for manufacturing.

Convective instabilities such as Tollmien-Schlichting (TS) waves may lead to the onset of laminar-turbulent transition, and therefore, it becomes crucial to correctly model their interaction with the above-mentioned surface irregularities. In the particular case of humps, a detailed analysis of how an incoming TS wave was affected by the presence of a two-dimensional hump on a flat plate was done experimentally by Boiko et al. ${ }^{1}$ Later, Dovgal and Kozlov $^{2}$ extended those studies by changing the shape of the hump and the frequency of the incoming waves. They were able to reduce by a factor of four the maximum amplitude reached by a single TS wave by placing a ramp downstream next to the originally rectangular hump. Some years later, Danabasoglu et al. ${ }^{3}$ reproduced the initial findings of Boiko et al. ${ }^{1}$ by using Direct Numerical Simulation (DNS). They also considered rectangular humps of different heights. They suggested that disturbances growing in the separation zone have an inviscid character and are controlled by the instability of the shear layer at the edge of the separation zone. Similarly, Liu and $\mathrm{Liu}^{4}$ also used DNS in order to reproduce the findings of Dovgal and Kozlov. ${ }^{2}$ However, in the work of Liu and Liu, ${ }^{4}$ the shape of the roughness was defined by a hyperbolic cosine function rather than a 
rectangular hump. They stated that the differences in the shape of the hump were the reason for the disagreement in instability growth rate found when comparing their DNS results with the experimental results of Dovgal and Kozlov. ${ }^{2}$ Instead, Masad and Iyer $^{5}$ used Linear Stability Theory (LST) for reproducing the results of Dovgal and Kozlov. ${ }^{2}$ Masad and Iyer ${ }^{5}$ extended their studies by also considering compressibility effects. They investigated the effects of height, length, location, and shape of the hump. However, they examined humps with rounded corners only. The transition location was defined by applying the $e^{N}$ transition criterion: the predicted transition location takes places at the point where the $N$-factor reaches the threshold value of 9 . Some years later, Wörner et al. ${ }^{6}$ applied DNS to study the effect of height and length on rectangular humps on a single TS wave in an incompressible flow. They included results for a smooth hump in their paper, but a detailed description of the shape of the smooth hump was not provided. Among the geometrical parameters considered (height, length, and shape), they identified the height of the hump as the geometrical parameter that has the strongest influence on the amplification of the incoming TS wave. However, because of the computational requirements of the DNS, their studies were limited to a very few geometries (six in total). More recently, Perraud et al. ${ }^{7}$ developed a model for predicting the increment in $\mathrm{N}$-factor due to the presence of a small bump based on numerical and experimental results. Their model considered smooth bumps of different shapes. However, they noticed that their model is restricted to cases where the height of the bumps is small enough that laminar separation does not occur. Similarly, Costantini et al. ${ }^{8}$ performed experimental investigations for smooth bumps, but also considering cases in which the height of the bump promotes laminar separation. Recently, Zhao et al. ${ }^{9}$ applied the HLNS equations to study the effect of two-dimensional rounded roughness on hypersonic boundary-layer transition.

Alternative to LST and DNS, Parabolized Stability Equations (PSE) have been successfully applied to study the growth of amplitude for convective instabilities in boundary-layer flows and, based on the $e^{N}$ methodology, to provide a reasonable estimate of the transition location. In the PSE approach, it is assumed that the streamwise variation of the laminar base flow is small over the characteristic wavelength of the instability mode. In the work of Thomas et al., ${ }^{10}$ they successfully applied PSE for studying the effect of small indentations, where the depth of the indentations was limited to 0.2 times the boundary-layer thickness. Similarly, Park and Park ${ }^{11}$ studied the effect of humps on incompressible flows using PSE. They were able to reproduce the DNS results of Wörner et al., ${ }^{6}$ but only for the smooth hump case. To the best of the authors' knowledge, there is no published comparison between PSE and DNS computations for the spatial evolution of convective instabilities in the presence of surface irregularities when the height of the irregularity is similar to the local boundary-layer displacement thickness or the shape of the surface irregularity contains sharp corners. To our understanding, in such cases, the streamwise gradients introduced by the surface irregularities violate the weakly nonparallel assumption of the PSE approach and, therefore, the PSE method is not suited for those cases.

The Local Scattering Approach (LSA), developed by Wu and Dong, ${ }^{12}$ is an asymptotic model based on the triple-deck theory that overcomes some of the limitations of the PSE method. However, an important constraint in this model is that LSA assumes that the surface irregularities remain in the lower-deck region ${ }^{12}$ of the boundary layer. In other words, the maximum allowable height of the irregularity is $h^{*} / \delta^{*}=\mathcal{O}\left(R e^{-1 / 8}\right)$, where $\delta^{*}$ is the displacement thickness of the smooth boundary layer and $R e$ is the Reynolds number $R e=U_{\infty}^{*} x_{c}^{*} / v_{\infty}^{*}$, with $U_{\infty}^{*}$ and $v_{\infty}^{*}$ denoting the velocity and kinetic viscosity of the oncoming stream, respectively. Dong and Zhang $^{13}$ applied LSA to study the effect of steps and humps on flat plates. However, their findings were limited to cases with rounded corners.

The AHLNS methodology was introduced by Guo et al. ${ }^{14}$ for studies on the generation of convective instabilities and their subsequent downstream development in laminar boundary layers by slowly varying streamwise properties. Franco and Hein ${ }^{15}$ developed it further and extended its range of applications to cases with localized large streamwise gradients, making use of its full potential. According to our understanding, when combined with PSE, it represents the most efficient tool for a detailed study of the linear interaction of convective instabilities with two-dimensional surface irregularities when the height of the surface irregularity is similar to the boundary-layer displacement thickness. It has been shown ${ }^{15}$ that AHLNS, combined with PSE, reproduces results found in the literature for the linear growth of convective instabilities in the presence of two-dimensional surface irregularities. This approach overcomes some of the inherent limitations present in techniques such as LST, PSE, and LSA. Moreover, it requires much less computational resources than DNS. This technique has been successfully applied already to study the effect of smooth humps and gaps on the spatial development of TS waves in a compressible regime. ${ }^{16}$ However, Ref. 16 was limited to variations in shape only, keeping the height and length fixed. Moreover, no rectangular-shaped humps were considered.

The present paper provides a systematic study of the effect of a single hump on the spatial development of TS waves in a compressible subsonic two-dimensional flow with zero pressure gradients. The height, length, and shape have been systematically varied in order to provide a clearer understanding of the flow physics involved and how those parameters influence the base flow and the linear growth of the oncoming TS waves. For each case, the spatial evolution of TS waves of 34 different frequencies was computed in order to calculate a detailed $N$-factor envelope curve. By applying the $e^{N}$ methodology, an expected transition location is established for each case. To the best of the authors' knowledge, there is no similar work published neither from experimental results nor from numerical computations.

\section{PROBLEM DESCRIPTION}

\section{A. Setup}

We numerically study the effect of a single surface roughness on boundary-layer transition of a two-dimensional laminar flow on a flat plate. All dimensional quantities are marked with an asterisk $*$. We place the origin of the Cartesian coordinate system at the leading edge of the plate, where $x^{*}, y^{*}$, and $z^{*}$ are the streamwise, normal, and spanwise coordinate components of the position vector $\mathbf{x}^{*}$, respectively. The freestream Mach number $M a_{\infty}$ is set to 0.5 for all cases. The shape of the surface roughness is defined 


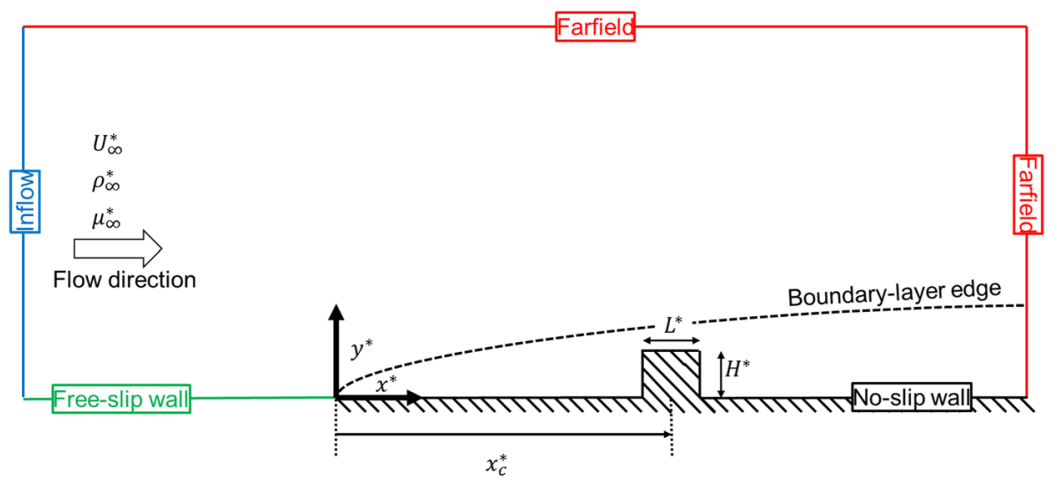

FIG. 1. Schematic representation of the computational domain defined for the present study. The labels at the edges of the domain indicate the types of boundary conditions used for the base flow computations.

by the following expression:

$$
y^{*}=H^{*} \exp \left(-\left(\frac{x^{*}-x_{c}^{*}}{L^{*} / 2}\right)^{2 m}\right), \quad \text { with } m=1,2,3,4, \ldots,
$$

where the parameter $H^{*}$ indicates the height of the hump and parameter $L^{*}$ its length. The center of the hump is placed at $x_{c}^{*}$. Figure 1 schematically shows the computational domain used in the present study, including the types of boundary conditions imposed for computing the laminar base flow. At the inflow, free-stream conditions are imposed. The sharp leading edge of the flat plate is represented by a change in boundary conditions at the lower wall (from free-slip to no-slip boundary conditions). The geometrical parameter $m$ will be varied from 1 to 4 , where the case $m=1$ corresponds to a Gaussian-shaped roughness. The rectangular shape can be considered as the limiting case when $m \rightarrow \infty$ (also examined in the present study). Geometrical quantities are nondimensionalized with $\delta^{*}$ (the compressible boundary-layer displacement thickness at the $x_{c}^{*}$ position for a flat plate at zero pressure gradient). The value of $\delta^{*}$ is given by the following expression (see Ref. 17):

$$
\delta^{*}=\int_{0}^{\infty}\left(1-\frac{\rho^{*} u^{*}}{\rho_{\infty}^{*} U_{\infty}^{*}}\right) d y^{*},
$$

where $\rho^{*}$ and $u^{*}$ stand for the density and streamwise velocity component, while $\rho_{\infty}^{*}$ and $U_{\infty}^{*}$ refer to density and velocity at the boundary-layer edge, respectively. The Reynolds number based on $\delta^{*}$ is defined as

$$
R e_{\delta^{*}}=\frac{\rho_{\infty}^{*} U_{\infty}^{*} \delta^{*}}{\mu_{\infty}^{*}},
$$

where $\mu_{\infty}^{*}$ denotes the dynamic viscosity at the boundary-layer edge. In the present study, the value of $R e_{\delta^{*}}$ is fixed to 1823 . As mentioned above, we will consider five values for the geometric parameter $m: 1,2,3,4$, and the rectangular-shaped case $r$, i.e., $m \rightarrow \infty$. For the nondimensional height $H$, three values will be examined here: $0.4,0.8$, and 1.2 . In the present paper, the height of the roughness will also be referred to as small $(H=0.4)$, medium $(H=0.8)$, and large $(H=1.2)$. The nondimensional length $L$ of the surface irregularity is fixed to 100 for small and medium roughnesses. For large roughnesses, three different values of $L$ will be considered: 50,
100 , and 150 . The combination of parameters $m, H$, and $L$ gives a total of 25 types of surface irregularities, which are summarized in Table I.

\section{B. AHLNS equations}

The boundary-layer instability analysis of the above-mentioned configuration will be done using the Adaptive Harmonic Linearized Navier Stokes (AHLNS) ${ }^{15}$ equations in combination with linear

TABLE I. Values of non-dimensional parameters $H, L$, and $m$ considered in the present study. The case $m=r$ refers to the rectangular-shaped humps. The last column indicates the maximum slope of the wall.

\begin{tabular}{lcccc}
\hline \hline Case name & $\mathrm{H}$ & $\mathrm{L}$ & $\mathrm{m}$ & Maximum slope $\left(^{\circ}\right)$ \\
\hline H4_m1 & 0.4 & 100 & 1 & 0.39 \\
H4_m2 & 0.4 & 100 & 2 & 0.70 \\
H4_m3 & 0.4 & 100 & 3 & 1.02 \\
H4_m4 & 0.4 & 100 & 4 & 1.36 \\
H4_r & 0.4 & 100 & $\mathrm{r}$ & 90 \\
H8_m1 & 0.8 & 100 & 1 & 0.79 \\
H8_m2 & 0.8 & 100 & 2 & 1.40 \\
H8_m3 & 0.8 & 100 & 3 & 2.04 \\
H8_m4 & 0.8 & 100 & 4 & 2.71 \\
H8_r & 0.8 & 100 & $\mathrm{r}$ & 90 \\
H12_m1 & 1.2 & 100 & 1 & 1.18 \\
H12_m2 & 1.2 & 100 & 2 & 2.08 \\
H12_m3 & 1.2 & 100 & 3 & 3.07 \\
H12_m4 & 1.2 & 100 & 4 & 4.07 \\
H12_r & 1.2 & 100 & $\mathrm{r}$ & 90 \\
H12_L50_m1 & 1.2 & 50 & 1 & 2.36 \\
H12_L50_m2 & 1.2 & 50 & 2 & 4.15 \\
H12_L50_m3 & 1.2 & 50 & 3 & 6.12 \\
H12_L50_m4 & 1.2 & 50 & 4 & 8.06 \\
H12_L50_r & 1.2 & 50 & $\mathrm{r}$ & 90 \\
H12_L150_m1 & 1.2 & 150 & 1 & 0.79 \\
H12_L150_m2 & 1.2 & 150 & 2 & 1.40 \\
H12_L150_m3 & 1.2 & 150 & 3 & 2.05 \\
H12_L150_m4 & 1.2 & 150 & 4 & 2.72 \\
H12_L150_r & 1.2 & 150 & $\mathrm{r}$ & 90 \\
\hline \hline
\end{tabular}


PSE. ${ }^{18}$ Here, a brief introduction to the AHLNS methodology is given. For a detailed description of this methodology, the readers are referred to the precursory work of Guo et al. ${ }^{14}$ or the more recent work of Franco and Hein ${ }^{15}$ (focused on the effect of surface imperfections on boundary-layer instabilities). For a complete explanation about the PSE methodology, the readers are referred to Bertolotti et al. ${ }^{19}$ and Herbert. ${ }^{20}$

The AHLNS equations are obtained from the compressible Navier-Stokes (NS) equations linearized for small disturbances. All flow and material quantities $\mathbf{q}$ are decomposed into a steady base flow $\overline{\mathbf{q}}$ plus an unsteady disturbance flow component $\tilde{\mathbf{q}}$, i.e.,

$$
\mathbf{q}(\mathbf{x}, t)=\overline{\mathbf{q}}(\mathbf{x})+\varepsilon \tilde{\mathbf{q}}(\mathbf{x}, t), \quad \varepsilon \ll 1,
$$

where $x, y$, and $z$ are the streamwise, normal, and spanwise coordinate components of the position vector $\mathbf{x}$, respectively. Here, $t$ represents time. This flow decomposition is introduced into the NS equations, then the base state solution is subtracted and products of disturbance quantities are neglected. To further simplify the analysis, it is assumed that the base flow is homogeneous in the spanwise $z$ direction. Moreover, disturbances are assumed to be periodic in time $t$ and in the spanwise $z$ direction. The adaptive approach is introduced here: the disturbance flow variables are divided into an amplitude function and a suitable, iteratively updated wave function, i.e.,

$$
\tilde{\mathbf{q}}(x, y, z, t)=\hat{\mathbf{q}}(x, y) e^{i \Theta},
$$

where the wave function is defined as

$$
\Theta=\int \alpha(x) d x+\beta z-\omega t
$$

Here, $\alpha, \beta$, and $\omega$ stand for streamwise wavenumber, spanwise wavenumber, and frequency of the disturbance, respectively. The physical disturbance is recovered from the real part of $\tilde{\mathbf{q}}$.

The major advantage of the adaptive approach is that it exploits the wave-like character of the convective instabilities in a similar fashion as in the PSE method. However, unlike the PSE approach, ${ }^{18}$ two aspects are crucial in this approach: First, the streamwise wavenumber $\alpha$ is allowed to rapidly vary in the streamwise direction. Second, the adaptive approach does not introduce any further simplification in the system of equations, i.e., all terms of the linearized Navier-Stokes (LNS) equations are kept.

Once the wave decomposition mentioned above is introduced into the LNS equations, the AHLNS equations are obtained. These equations can be written in matrix form

$$
\mathbf{A} \hat{\mathbf{q}}+\mathbf{B} \frac{\partial \hat{\mathbf{q}}}{\partial y}+\mathbf{C} \frac{\partial^{2} \hat{\mathbf{q}}}{\partial y^{2}}+\mathbf{D} \frac{\partial \hat{\mathbf{q}}}{\partial x}+\mathbf{E} \frac{\partial^{2} \hat{\mathbf{q}}}{\partial x \partial y}+\mathbf{F} \frac{\partial^{2} \hat{\mathbf{q}}}{\partial x^{2}}=0
$$

where $\hat{\mathbf{q}}=(\hat{u}, \hat{v}, \hat{w}, \hat{T}, \hat{p})$. Here, $p$ and $T$ stand for pressure and temperature, while $u, v$, and $w$ are the streamwise, normal, and spanwise components of the velocity vector $\mathbf{u}$, respectively. The coefficients $\mathbf{A}-\mathbf{F}$ are $5 \times 5$ matrices, which contain base flow quantities, real-valued parameters $\beta$ and $\omega$, and the complex-valued wavenumber $\alpha$. The disturbance field is subjected to the following boundary conditions at the wall and at the farfield:

$$
\begin{gathered}
\hat{u}=\hat{v}=\hat{w}=\hat{T}=0 \quad \text { at } \quad y=0 \\
\hat{u}=\hat{v}=\hat{w}=\hat{T}=\hat{p} \rightarrow 0 \quad \text { as } \quad y \rightarrow \infty .
\end{gathered}
$$

At the inflow, prescribed values for all five quantities are imposed. At the outflow, disturbance quantities are extrapolated from inner points. Once the boundary conditions are incorporated into the system of equations, Eq. (7), and after discretization, a system of linear algebraic equations of the form $\mathbf{L} \hat{\mathbf{q}}=\mathbf{b}$ is reached, where vector $\mathbf{b}$ collects the information on the inflow boundary condition.

The streamwise wavenumber distribution $\alpha(x)$ is adjusted iteratively in a similar fashion as in the PSE approach, ${ }^{20}$ i.e.,

$$
\alpha_{\text {new }}=\alpha_{\text {old }}-i \frac{\int_{0}^{\infty}\left(\hat{\mathbf{q}}^{\dagger} \frac{\partial \hat{\mathbf{q}}}{\partial x}\right) d y}{\int_{0}^{\infty}\left(\|\hat{\mathbf{q}}\|^{2}\right) d y},
$$

with superscript ${ }^{\dagger}$ referring to the complex conjugate. The adjustment of $\alpha(x)$ is repeated iteratively, until $\alpha(x)$ can be considered as converged.

In order to extend the capabilities of the AHLNS approach for studying surface irregularities of any particular shape, two features have been implemented: ${ }^{15}$ First, a multi-block technique is used in cases where the surface irregularity contains sharp corners (i.e., rectangular humps). Second, a coordinate transformation is applied in order to transform the physical domain $(x, y)$ into the equally spaced computational domain $(\xi, \eta)$. If the multi-block technique is used, then the coordinate transformation is applied in each block individually (in order to avoid singularities at the sharp corners). At the interface between two neighboring blocks, the amplitude functions and their first derivatives normal to the block boundaries have to match at both sides of the interface. The spatial derivatives in this computational domain are discretized using a 4th-order finitedifference scheme in both $(\xi, \eta)$ directions. Based on our experience, we did not find any significant benefits from using discretization schemes of even higher order.

\section{Multi-zonal approach}

Figure 2 illustrates a typical example of a wall-bounded flow in the presence of a local surface irregularity (in this case, a rectangular hump on a flat plate) and indicates the region where AHLNS equations are applied in a multi-zonal approach. Relatively far away from the surface irregularity, the streamwise variations of the laminar base flow $\partial \bar{q} / \partial x$ and amplitude functions $\partial \hat{q} / \partial x$ are barely affected by the presence of the irregularity. These variations are relatively

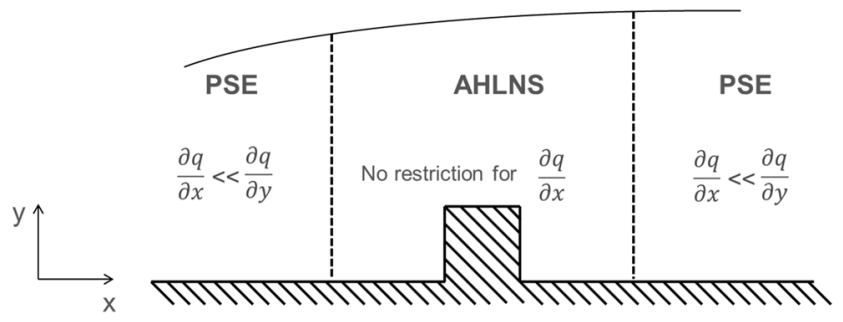

FIG. 2. Sketch of the multi-zonal technique for boundary-layer instability analysis in the presence of surface irregularities. The vertical dashed lines represent the inflow and outflow locations for AHLNS computations. Magnitude $q$ holds for both base flow quantities $\bar{q}$ and amplitude function of the disturbances $\hat{q}$. Flow direction is from left to right. 
small (compared with normal changes $\partial \bar{q} / \partial y$ and $\partial \hat{q} / \partial y$, respectively), and the assumptions made for PSE are valid. However, in the vicinity of the surface irregularity, the streamwise gradients may be of the same order as the normal gradients. In this region, the use of PSE could be questionable and, therefore, the AHLNS equations are applied. At the inflow location of the AHLNS zone, the amplitude functions $\hat{q}_{P S E}$ and streamwise wavenumber $\alpha_{P S E}$ provided by PSE are taken as inflow boundary conditions for AHLNS. At the outflow location of the AHLNS zone, the amplitude functions $\hat{q}_{A H L N S}$ and streamwise wavenumber $\alpha_{A H L N S}$ provided by AHLNS can be used as inflow conditions for a subsequent PSE computation. The inflow and outflow boundaries of the AHLNS domain are placed at a certain distance from the roughness, where the assumption of a slow streamwise variation of the laminar base flow and of the amplitude functions is still valid.

In the present paper, results obtained using this multi-zonal approach will be labeled AHLNS results. But actually, the results shown in this work are obtained by the multi-zonal combination of PSE and AHLNS methodologies described above.

\section{Growth rate and $\mathbf{N}$-factor envelope}

The physical growth rate $\sigma$ of an arbitrary disturbance quantity $\zeta$ is defined as

$$
\sigma_{\zeta}=-\alpha_{i}+\operatorname{Real}\left(\frac{1}{\zeta} \frac{\partial \zeta}{\partial x}\right)
$$

where the first rhs term is the contribution from the wave part of the disturbance [Eq. (5)]. The second term is the correction due to changes in the amplitude function. In the present paper, $\zeta$ is taken to be the streamwise velocity component $\hat{u}$ at the $y$ location, where $\hat{u}$ reaches its maximum value. The $n$-factor, which measures the accumulated growth of the disturbances, is computed as

$$
n=\int_{x_{s}}^{x} \sigma_{\hat{u}} d x
$$

where $x_{s}$ denotes the streamwise position where the disturbance starts to grow. Similar to the work of Ragab and Nayfeh, ${ }^{21}$ the $n$ factor is set to zero if during the course of the integration, the value of $n$ becomes negative. Each $n$-factor curve is computed for a single wave defined by a particular nondimensional frequency $F$ and spanwise wavenumber $\beta$. The nondimensional frequency $F$ is defined as

$$
F=2 \pi f^{*} \frac{\mu_{\infty}^{*}}{\rho_{\infty}^{*}\left(U_{\infty}^{*}\right)^{2}} \times 10^{6},
$$

where $f^{*}$ is the physical frequency of the disturbances. The envelope of these curves is called the $N$-factor envelope, following the definition given by Arnal. ${ }^{22}$

\section{E. Validation of the AHLNS methodology}

The AHLNS methodology has been validated already (see Ref. 15) for the spatial evolution of TS waves present in wall-bounded incompressible flows. Here, we include a case found in the work of Edelmann ${ }^{23}$ where the Mach number was set to 0.8 . The geometrical configuration of this case corresponds to a rectangular forwardfacing step, placed on a flat plate at $x_{c}^{*}=1 \mathrm{~m}$, with a unit Reynolds number of $R e_{u}=2.45 \times 10^{6} \mathrm{~m}^{-1}$ and a Reynolds number $R e_{H}$ based on the step height of $R e_{H}=1320$. This configuration is very similar to that of Fig. 1. However, in this section, we are considering a rectangular forward-facing step instead of a rectangular hump. The reduced frequency $F$ selected for this comparison was 21.875. Figure 3 compares the $n$-factor evolution of a TS wave computed by AHLNS with the results of Edelmann ${ }^{23}$ using DNS. This case is very useful not only to show the very good agreement between AHLNS and DNS but also to illustrate some differences between both methodologies. In the work of Edelmann, ${ }^{23}$ the number of grid points used in the DNS computations is not mentioned explicitly. However, we can still illustrate the differences in terms of computational resources needed for this validation case. Edelmann stated that, for a single run, 6144 processors were used at the High Performance Computed Center Stuttgart (HLRS) for the DNS. Depending on the finite-difference scheme he used, each run took between $103 \mathrm{~s}$ (explicit 8th-order finite differences) and $352 \mathrm{~s}$ (compact 6th-order finite differences). On the other hand, for this validation test, our AHLNS computation was done using a single work-station with four cores and the result was obtained after $300 \mathrm{~s}$ (explicit 4th-order finite differences). We used a mesh with $500 \times 200$ points in streamwise and normal directions, respectively.

We believe that there are three main reasons for the different amount of computational effort needed in both methodologies:

- The AHLNS method (like PSE) assumes that instabilities are convective in nature and can be expressed in a normalmode form (i.e., the wave-like character of the disturbances is exploited). However, in DNS (like LNS or HLNS) no assumptions are made regarding the nature of the instabilities in the streamwise direction.

- The effect of the step on the development of the TS wave is very localized. For this reason, we apply AHLNS in a small region in the vicinity of the step (i.e., $x^{*} \in[0.9,1.1] \mathrm{m}$ ), while we make use of PSE in the rest of the plate. The DNS used

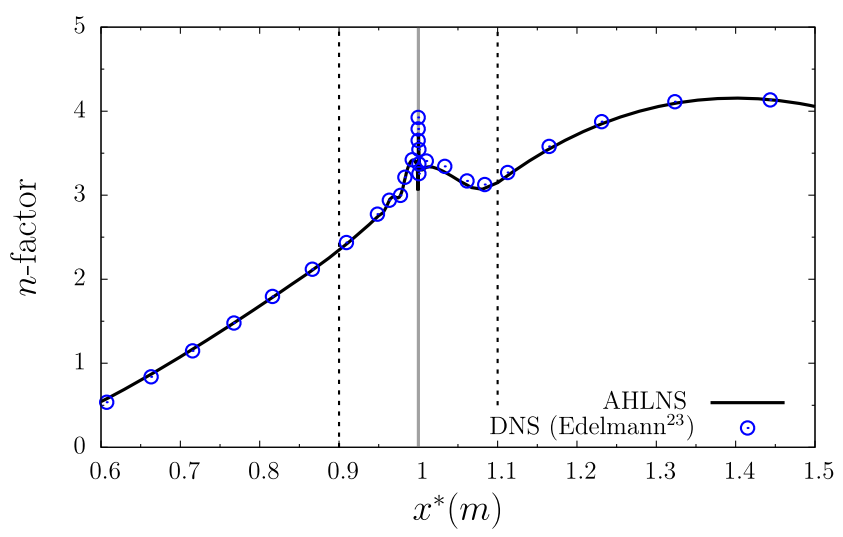

FIG. 3. $n$-factor distribution for an incoming TS wave of reduced frequency $F$ $=21.875$ along a flat plate in the presence of a rectangular forward-facing step. The gray line represents the location of the step. The two vertical dashed lines, placed at $x^{*}=0.9 \mathrm{~m}$ and $x^{*}=1.1 \mathrm{~m}$, indicate the position where the interfaces between PSE and AHLNS methods are placed. DNS data courtesy of C. A. Edelmann, "Influence of forward-facing steps on laminar-turbulent transition," Ph.D. dissertation (University of Stuttgart, 2014). 
by Edelmann does not apply this multi-zonal approach, and therefore, the whole domain is computed at once.

- The AHLNS approach (like PSE) assumes that disturbances are periodic in time. This assumption is not included in the DNS approach, and therefore, a time-integration scheme is used in the DNS solver. ${ }^{23}$ In order to achieve a converged time-periodic solution, Edelmann claimed that up to 20000 time steps were required for the DNS computations.

\section{NUMERICAL RESULTS}

\section{A. Base flow computations}

\section{Approach}

The laminar steady two-dimensional flow on a flat plate in the presence of a hump was computed numerically. We used the compressible Navier-Stokes solver TAU, ${ }^{24}$ developed at DLR. Grids were generated using the structured grid generator MEGACADS, also developed at DLR. A schematic representation of the computational domain and the boundary conditions imposed for computing the base flows is depicted in Fig. 1. Farfield boundary conditions were imposed sufficiently far from the surface irregularity (about $2500 \delta^{*}$ in both streamwise and normal directions). Simulations were run until the variations in lift and drag coefficients within last 40 iterations were smaller than $10^{-7}$ and $3 \times 10^{-8}$, respectively.

\section{Grid convergence}

Three sets of grids were used to verify the grid-independence of the base flow results. In all sets of grids, points were clustered near the walls, where the largest variations of flow quantities are expected. The first set of grids is referred to as coarse. These grids have about 700 and 150 points in streamwise and normal directions, respectively. The number of points can vary slightly in the normal direction when the value of $H$ is changed (in order to keep the same grid spacing close to the wall). Another set of grids, named medium, was also used to compute the base flows. In this second set of grids, the number of points was increased in both directions: about 1100 and 225 points in streamwise and normal directions, respectively.
The number of points within the boundary layer is about $70 \%$ of the total number of points in the normal direction for all meshes considered in the present work. Finally, the third group of grids, called fine, has about 1500 and 300 points in streamwise and normal directions, respectively.

Figure 4 shows the results of the grid convergence study for the case H12_r. Depicted in this figure is the dividing line $\bar{u}=0$ that limits the region where the reverse flow $(\bar{u}<0)$ is present. For this case, three recirculation areas appear: one in front of the hump, one on top of the hump [both represented in Fig. 4(a)], and a large recirculation area after the hump [shown in Fig. 4(b)]. The size of these recirculation regions is only slightly smaller in the case of coarse grid, but identical for medium and fine meshes. Therefore, the grid-independence of the base flow computations is demonstrated. The base flows for all cases considered in this work are stationary. The largest peak-recirculation velocity found is smaller than $4 \%$. Therefore, we are not expecting that global instabilities, in the sense of self-excitation of the base flow, play any role in the stability analysis (see Ref. 25). Figure 5 shows the results of the grid convergence study for the case H12_m4 based on the skin-friction coefficient $c_{f}$ and nondimensional displacement thickness $\delta$ distribution. The value of $c_{f}$ is given by the following expression (see Ref. 17):

$$
c_{f}=\frac{\left(\mu \frac{\partial u}{\partial y}\right)_{y=0}}{\frac{1}{2} \rho_{\infty}^{*}\left(U_{\infty}^{*}\right)^{2}} .
$$

Results for the flat plate case have been added for comparison. Similar to the above-mentioned case H12_r, the results show that the base flows are grid-independent for medium and fine grids. Figure 5 illustrates another fact that has to be pointed out: The presence of the hump affects strongly the base flow characteristics, but this effect is limited to the vicinity of the hump. In other words, at a certain distance of the hump, the base flow properties in the streamwise direction change slowly (compared with variations in normal direction), similar to the flat plate case. This circumstance (localized influence of the surface irregularity) permits us to apply the multizonal approach described in Sec. II C for the subsequent stability analysis. As a result of the grid-convergence study, the base flow
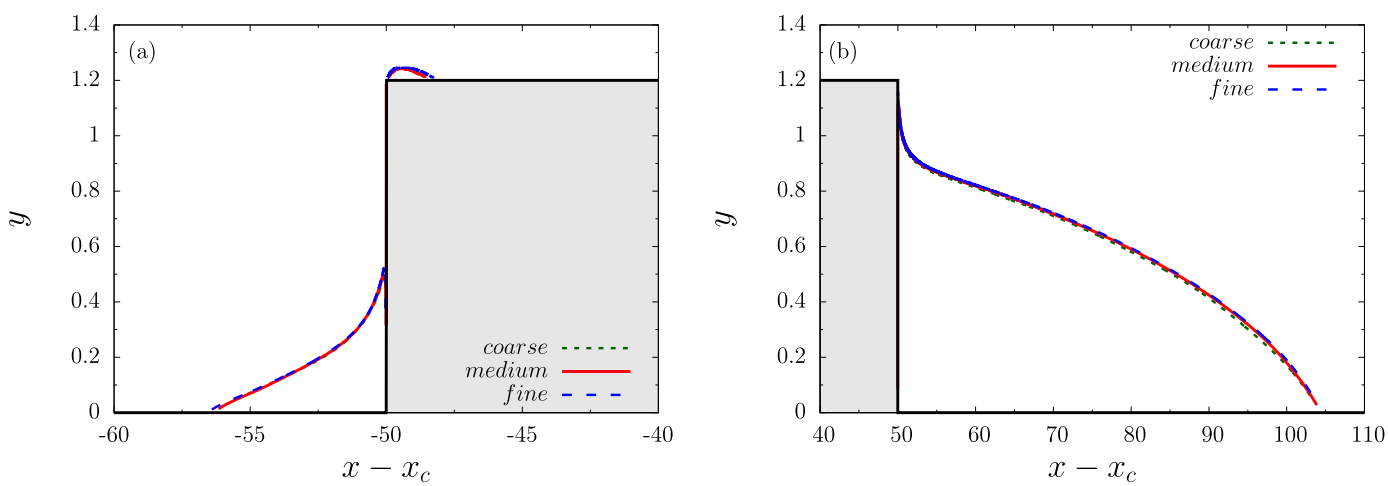

FIG. 4. Base flow grid convergence study for case H12_r. The green dashed lines (coarse), continuous red lines (medium), and long-dashed blue lines (fine) represent the points in the base flow where $\bar{u}=0$, upstream (a) and downstream (b) of the hump. The gray area represents the hump. Flow direction is from left to right. Axes are not to scale. 

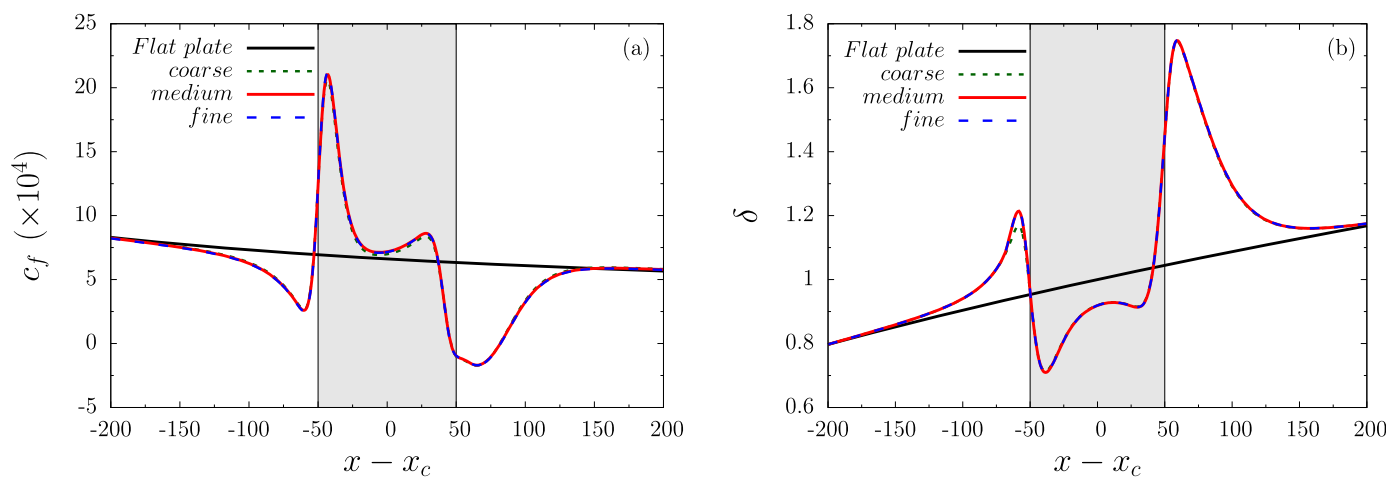

FIG. 5. Base flow grid convergence study for case $H 12 \_m 4$, based on skin-friction coefficient $c_{f}$ (a) and nondimensional displacement thickness $\delta$ (b). The gray area indicates the position of the hump.

computed with meshes of the set medium will be used for stability analysis.

\section{Base flow results}

The base flow pressure $\bar{p}$ is made nondimensional by twice the incoming dynamic pressure, i.e., $\rho_{\infty}^{*}\left(U_{\infty}^{*}\right)^{2}$. Figure 6 compares the contours of $\bar{p}$ and streamlines for several cases. For small $(H=0.4)$ roughnesses [Figs. $6(\mathrm{a})-6(\mathrm{c})$ ], the flow field exhibits little variations in the three cases considered. Only in the rectangular case $H 4 \_r$ [Fig. 6(c)], there is a tiny separation bubble at the downstream side of the hump. The streamwise pressure gradients at the leading and trailing edges of the humps are more intense as the humps tend

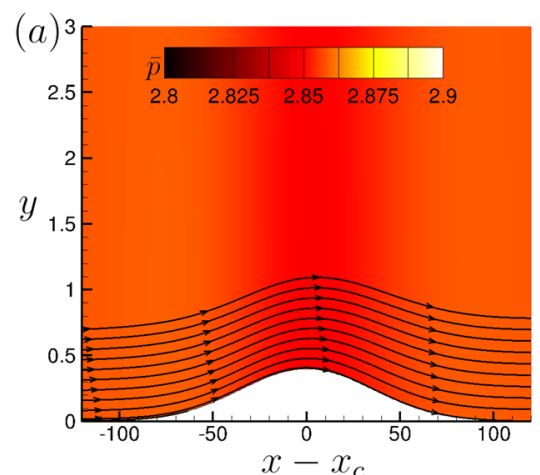

Case H4_m 1

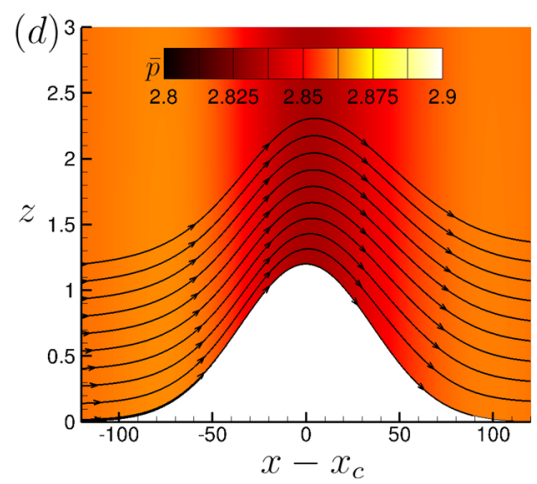

Case $H 12 \_m 1$

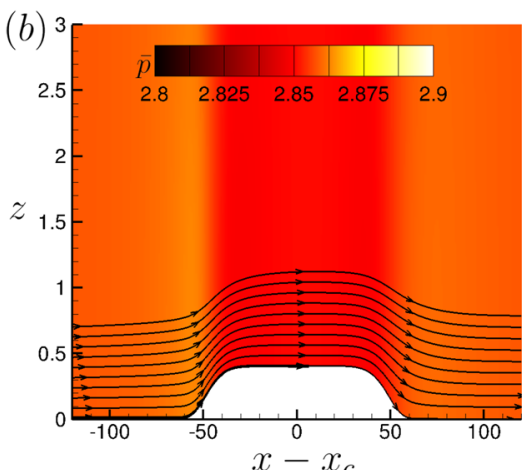

Case $H 4 \_m 4$

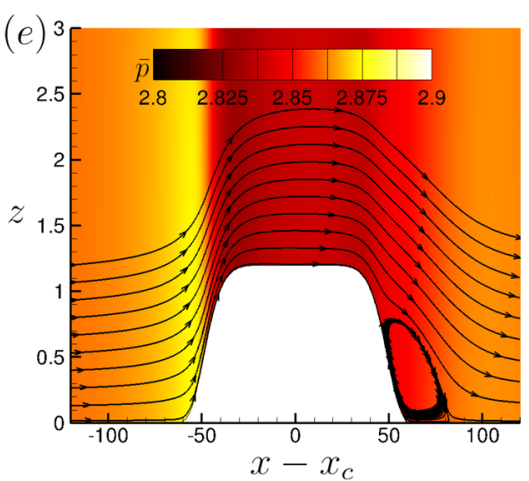

Case $H 12 \_m 4$

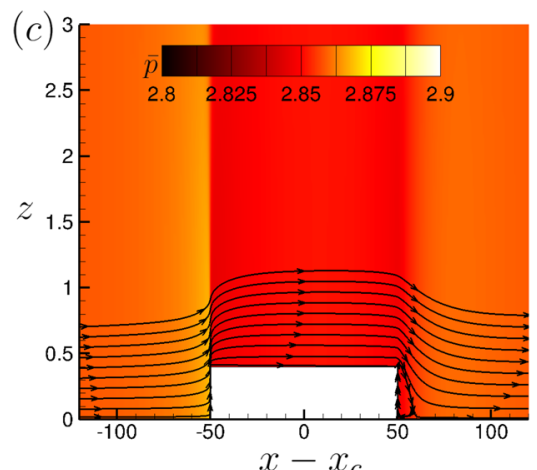

Case $H 4$

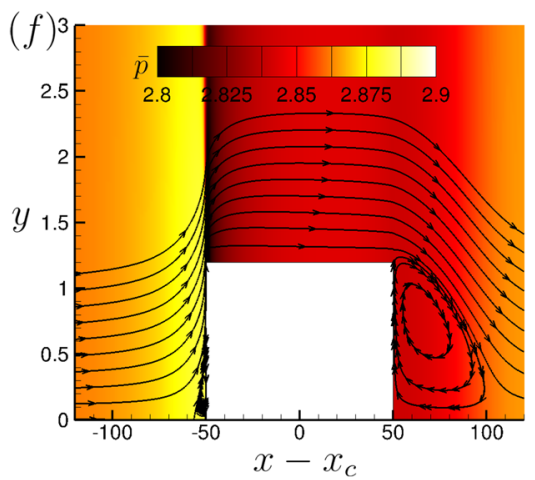

Case $H 12 \_r$

FIG. 6. Pressure $\bar{p}$ contours and streamlines for small $(H=0.4)$ and large $(H=1.2)$ humps in cases $m=1$ [labels $(\mathrm{a})$ and $(\mathrm{d})], 4$ [labels (b) and (e)], and $r$ [labels $(\mathrm{c})$ and (f)], respectively. Flow direction is from left to right. Axes are not to scale. 
to the rectangular shape (i.e., the value of $m$ increases). For large $(H=1.2)$ roughnesses [Figs. $6(\mathrm{~d})-6(\mathrm{f})$ ], variations in the flow field are more relevant. The flow in case H12_m1 [Fig. 6(d)] remains attached, although the pressure gradients are more pronounced than in case $H 4 \_m 1$. For case H12_m4 [Fig. 6(e)], a separation bubble appears at the trailing edge of the hump. The length of this bubble (in the $x$-direction) is approximately one quarter of the length $L$ of the hump. The last case considered here, namely, H12_r [Fig. 6(f)], exhibits a large separation bubble downstream of the hump (approximately one half of the length $L$ in the streamwise direction). The disturbance in the base flow provoked by the presence of the roughness is limited to the vicinity of the hump. At a certain distance from the hump, the flow field recovers the key characteristics of the undisturbed flow field (i.e., small variations of flow magnitudes in the streamwise direction). This fact, highlighted above in Fig. 5, assures that the multi-zonal approach proposed in Sec. II C is perfectly suited for this configuration.

\section{B. Instability analysis}

The computation of the spatial development of a broadband spectrum of TS waves (each one computed individually and defined by its frequency $\mathrm{F}$ and spanwise wavenumber $\beta$ ) is described in this section. The spatial growth in amplitude of each TS wave is collected in the corresponding $n$-factor curve (defined in Sec. II D).

The instability analysis is computed in the domain $\left(x-x_{c}\right) \epsilon$ $[-500,2000]$. An initial investigation performed on a flat plate using PSE revealed that two-dimensional $(\beta=0)$ TS waves of frequencies $F \in[20,75]$ produce the highest $n$-factor curves in the domain mentioned above. When a surface irregularity is present, the multi-zonal approach described in Sec. II C is applied. The interfaces between PSE and AHLNS are placed at $\left(x-x_{c}\right)= \pm 165$, sufficiently far away from the recirculation regions (see Fig. 6). The following results were obtained considering incoming two-dimensional TS waves $(\beta=0)$ only. The effect of humps on three-dimensional waves $(\beta \neq 0)$ will be discussed in Sec. III G.

\section{Grid convergence}

The presence of sharp corners increases significantly the number of points required for a correct representation of the TS waves in the vicinity of the hump. This is the reason to select two humps (H8_r and H8_m4) to prove that the instability results presented here are grid-independent. For rounded humps, the reference grid is made of 100 points in the streamwise direction and 100 points in the normal direction. However, for cases where sharp corners are present the reference grid is made of $500 \times 300$ points in streamwise and normal directions, respectively. Moreover, we will refer here only to the number of points used for the AHLNS computations $\left\{\right.$ i.e., in the domain $\left.\left(x-x_{c}\right) \in[-165,165]\right\}$. For each hump, the number of points for the stability analysis is increased by $40 \%$ in each direction independently with respect to the corresponding reference grid. Figure 7 reports the results of this investigation for a single TS wave of frequency $F=48$.

\section{Effect of hump height and shape}

Figure 8 illustrates how the $N$-factor envelope curve is built for each hump (here, the case $H 4 \_m 1$ is considered). A total of

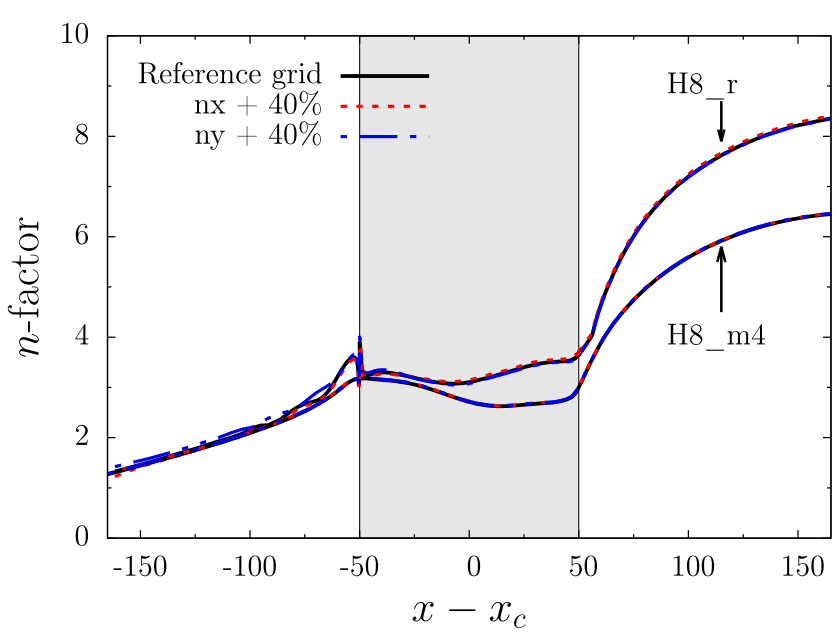

FIG. 7. Stability analysis grid convergence study for cases $H 8 \_r$ and $H 8 \_m 4$. Black solid lines represent AHLNS computations using the reference grid. For rectangular humps, this reference grid consists of $(n x, n y)=(500,300)$ points. For smooth humps, the reference grid consists of $(n x, n y)=(100,100)$ points. The results marked by red dashed and blue dashed-dotted lines were obtained by increasing the number of points in streamwise and normal directions by $40 \%$, respectively. The gray area indicates the position of the hump.

34 TS waves, with frequencies in the range between $F=20$ and $F=75$, are computed. At each streamwise location, the maximum $n$-factor value for all frequencies computed is taken as the corresponding $N$-factor at that particular location. This process is repeated for the 25 cases considered in the present paper (see Table I). The results will be presented in Secs. III C 1-III C 3 by grouping the different roughnesses according to their height (small, medium, and large).

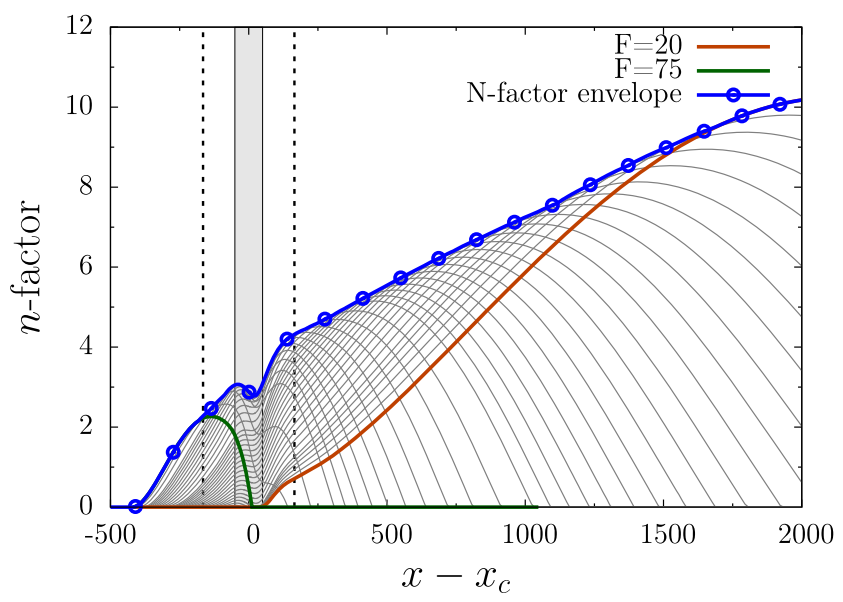

FIG. 8. Definition of the $N$-factor envelope curve (blue line with circles) for the case H4_m1. A total of 34 TS waves (gray lines) were computed. The smallest $(F=20)$ and largest $(F=75)$ frequencies considered have been highlighted for simplicity. Vertical dashed lines indicate the location of the interfaces between PSE and AHLNS methodologies. The gray area represents the position of the hump. 


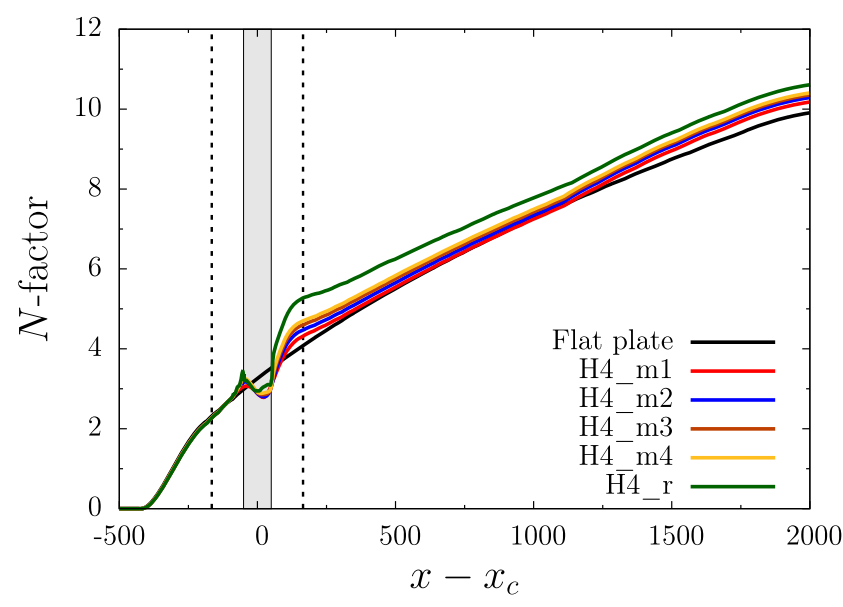

FIG. 9. $N$-factor envelope curves for small humps $(H=0.4)$. Vertical dashed lines indicate the location of the interfaces between PSE and AHLNS methodologies. The gray area represents the position of the hump.

\section{Small humps}

Figure 9 compares the $N$-factor envelope curves when small humps $(H=0.4)$ are considered. The spatial variation of the $N$-factor curves is similar for all five humps analyzed here. At the end of the domain (i.e., $x=x_{c}+2000$ ), the $N$-factor increases from 9.9 (flat plate) to 10.6 (case $H 4 \_r$ ) and 10.4 (case $H 4 \_m 4$ ). In the vicinity of the humps, the development of the TS waves is driven by the pressure gradients that the presence of the hump introduces into the flow field. It is well known (see Ref. 26) that an acceleration in the flow usually has a stabilizing effect. Figure 10 shows the nondimensional pressure distribution at the wall $\bar{p}_{\text {wall }}$ for three values of the shape factor $m: 1,4$, and rectangular. The flat plate case is also considered for comparison. In these cases, in the leading region of the hump $\left(x-x_{c} \approx-50\right)$, there is an initial deceleration of the flow followed

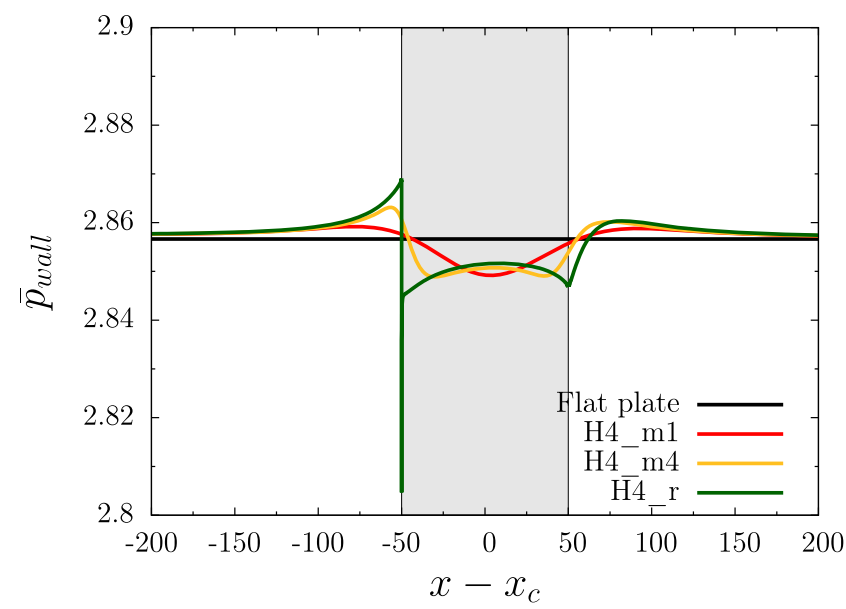

FIG. 10. Pressure distribution at the wall $\bar{p}_{\text {wall }}$ for small humps $(H=0.4)$ in cases $m=1$ (red line), $m=4$ (yellow line), and $m=r$ (green line). The flat plate case (black line) is included for comparison. The gray area represents the position of the hump. by a rapid acceleration. This effect explains why on the upstream side of the hump, there is a small increase in the $N$-factor curves followed by a reduction. In the rear part of the hump $\left(x-x_{c} \approx 50\right)$, the pressure distribution indicates that a rapid deceleration of the flow is followed by a less pronounced acceleration. The strong deceleration is translated into a rapid increase in the $N$-factor curves. The effect of reducing the smoothness of the hump (increasing $m$ ) provokes that the pressure variations concentrate at the borders of the hump. Downstream of the hump, where the zero pressure gradient is recovered, the $\mathrm{N}$-factor curves grow almost parallel to the flat plate case.

\section{Medium humps}

Figure 11 shows the $N$-factor envelope curves when medium humps $(H=0.8)$ are considered. At the end of the domain (i.e., $\left.x=x_{c}+2000\right)$, the $N$-factor is 12.5 for case $H 8 \_r$ and 11.5 for case $H 8 \_m 4$. The behavior of the $N$-factor in the vicinity of the humps is similar as described previously for small humps. However, since the height of the humps $H$ considered here is twice that of the small humps, the pressure gradients around the hump are more intense (see Fig. 12). Once the zero pressure gradient region is reached, the $N$-factor curves grow parallel to the flat plate case as expected.

\section{Large humps}

In this section, the results of the investigation for large humps $(H=1.2)$ are described. For simplicity, only the cases where the length $L$ is 100 are presented here. Variations of the parameter $L$ will be discussed in Sec. III D. Figure 13 plots the amplification curves for the five humps considered here. At the end of the domain (i.e., $x=x_{c}+2000$ ), the $N$-factor is 14.4 for case $H 12 \_r$ and 12.9 for case H12_m4. The pressure gradients (Fig. 14) follow a similar behavior as it was already described for the previous cases. Readers can observe [Fig. 13(b)] that differences between the rectangular hump (case $H 12 \_r$ ) and rounded humps are especially

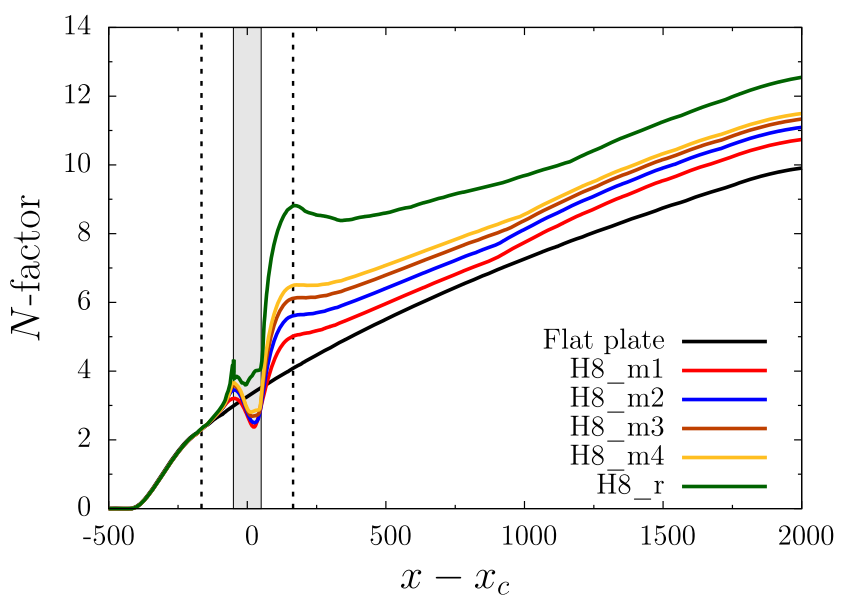

FIG. 11. $N$-factor envelope curves for medium humps $(H=0.8)$. Vertical dashed lines indicate the location of the interfaces between PSE and AHLNS methodologies. The gray area represents the position of the hump. 


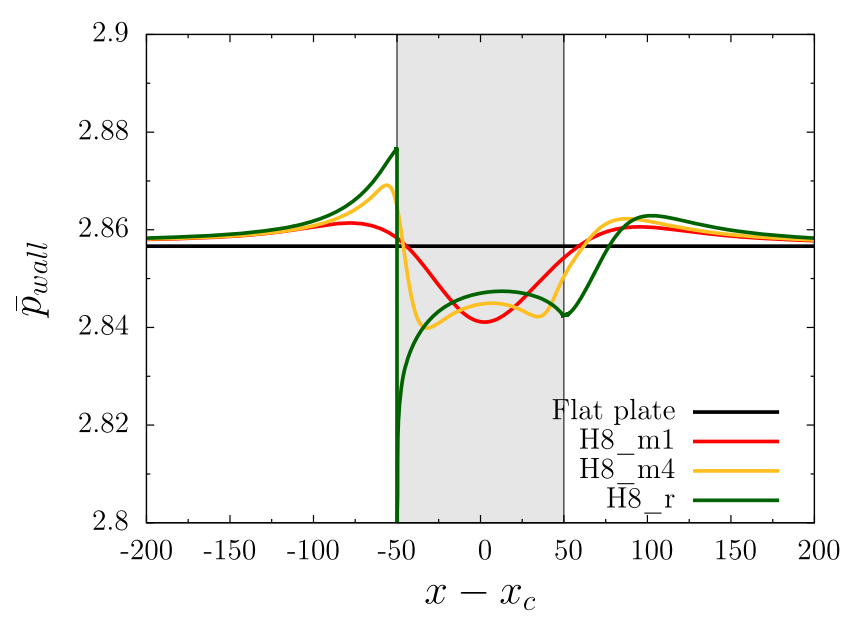

FIG. 12. Pressure distribution at the wall $\bar{p}_{\text {wall }}$ for medium humps $(H=0.8)$ in cases $m=1$ (red line), $m=4$ (yellow line), and $m=r$ (green line). The flat plate case (black line) is included for comparison. The gray area represents the position of the hump.

noticeable in the hump region. This effect indicates that humps with rounded edges alleviate the intensity of the pressure gradients around the hump, and therefore, they reduce the rapid variations of the $\mathrm{N}$-factor curve present in the rectangular-shaped case. As a result, the effect of the smooth-shaped humps is a significant reduction in terms of $\mathrm{N}$-factor compared with the rectangular case.

It is also important to note that the presence of reverse flow increases significantly the growth of the TS waves (see Ref. 27). Therefore, the existence and size of a recirculation bubble may play a significant role in the evolution of the $\mathrm{N}$-factor. As it was mentioned before [Figs. 6(d)-6(f)], at the trailing edge of the hump, the size of the recirculation bubble strongly depends on the shape of the hump. This finding also contributes to the understanding of how the shape of the hump affects the evolution of the $N$-factor.

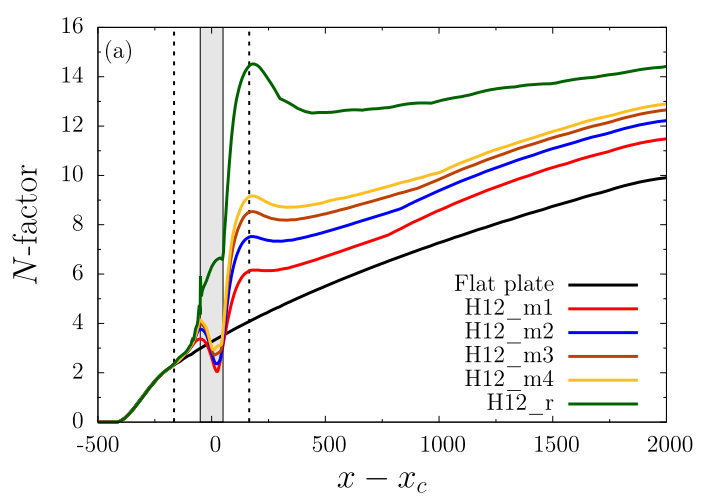

Complete domain $\left(x-x_{c}\right) \in[-500,2000]$

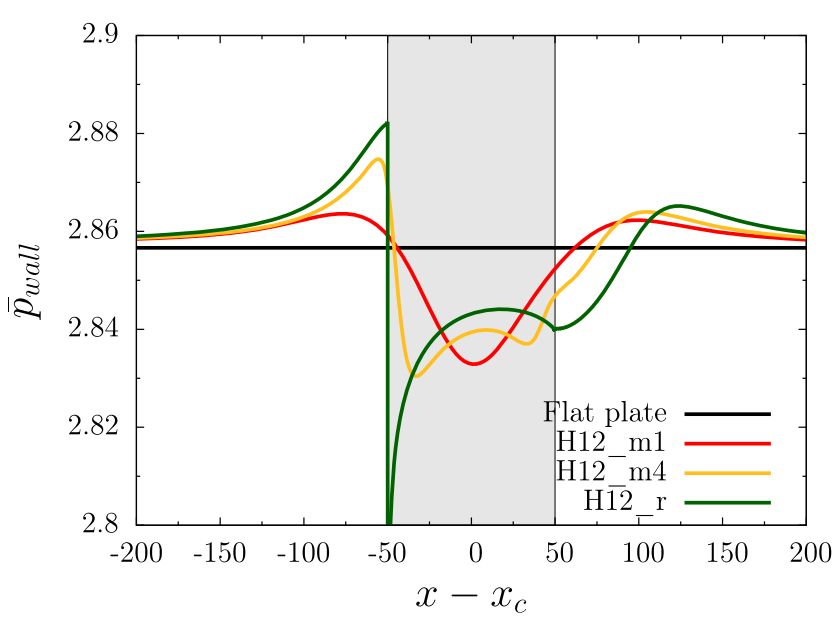

FIG. 14. Pressure distribution at the wall $\bar{p}_{\text {wall }}$ for large humps $(H=1.2)$ in cases $m=1$ (red line), $m=4$ (yellow line), and $m=r$ (green line). The flat plate case (black line) is included for comparison. The gray area represents the position of the hump.

\section{Effect of hump length}

For large humps $(H=1.2)$, the effect of the length $L$ is studied. Figure 15 shows the $N$-factor curves when the length is fixed to 50 . A comparison with the previous case $L=100$ indicates that the $N$ factor curve for the rectangular-shaped case $\left(H 12 \_L 50 \_r\right)$ is similar to case H12_r (Fig. 13). However, the $N$-factor values for rounded humps are significantly higher when the length $L$ is reduced. The reason for this behavior can be found again in the pressure distribution $\bar{p}_{\text {wall }}$ (Fig. 16). Reducing the length $L$ of the hump provokes that the pressure gradients around the rounded humps are significantly increased, resulting in an overall increase in the $N$-factor curves for the rounded humps.

Consequently, when the length of the hump is increased ( $L$ $=150$ ) the major effect is an overall reduction in the $N$-factor curves for rounded humps (compared with the cases $L=100$ ). However, the

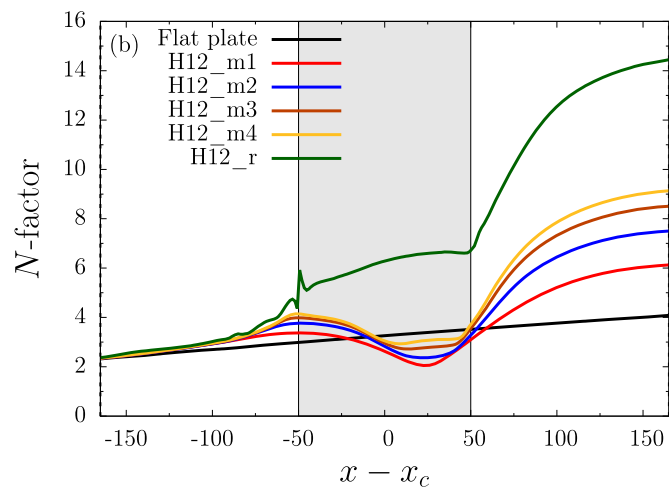

Detailed view $\left(x-x_{c}\right) \in[-165,165]$

FIG. 13. $N$-factor envelope curves for large humps $(H=1.2)$ and $L=100$, for the complete domain (a), and a detailed view in the vicinity of the hump (b). Vertical dashed lines indicate the location of the interfaces between PSE and AHLNS methodologies. The gray area represents the position of the hump. 


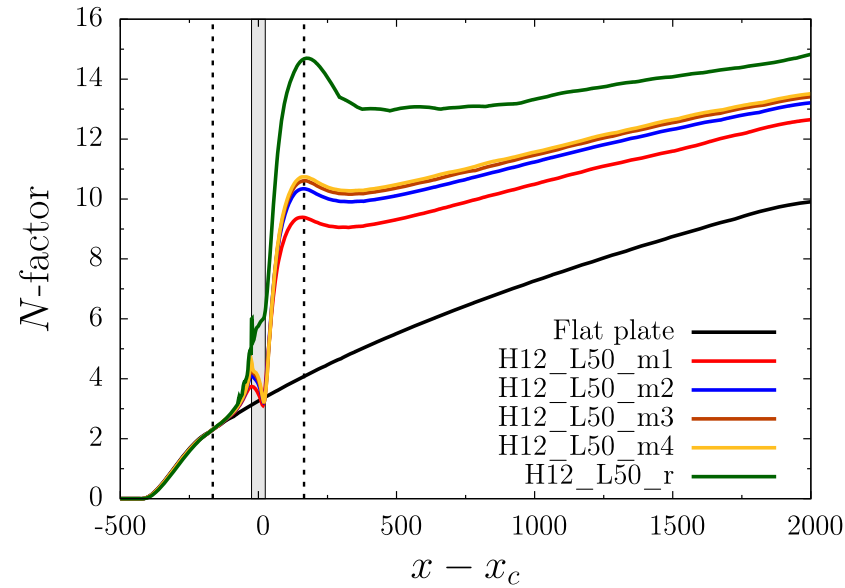

FIG. 15. $N$-factor envelope curves for large humps $(H=1.2)$ and $L=50$. Verti$\mathrm{cal}$ dashed lines indicate the location of the interfaces between PSE and AHLNS methodologies. The gray area represents the position of the hump.

$\mathrm{N}$-factor curve for the rectangular-shaped hump is barely affected by this increase in length (Fig. 17). The pressure distribution at the wall $\bar{p}_{\text {wall }}$ (Fig. 18) confirms these findings.

\section{E. Prediction of transition location}

The results of the linear instability analysis, together with the $e^{N}$ methodology, can lead to a predicted transition location $x_{t r}$. Without having any further information, it is very common ${ }^{5}$ to set the expected transition location $x_{t r}$ as the closest point to the leading edge where the amplification curves reach the threshold value of $N=9$. Therefore, starting upstream of the roughness location, we follow the $N$-factor curve downstream until it reaches this threshold value. The corresponding streamwise location is defined as the

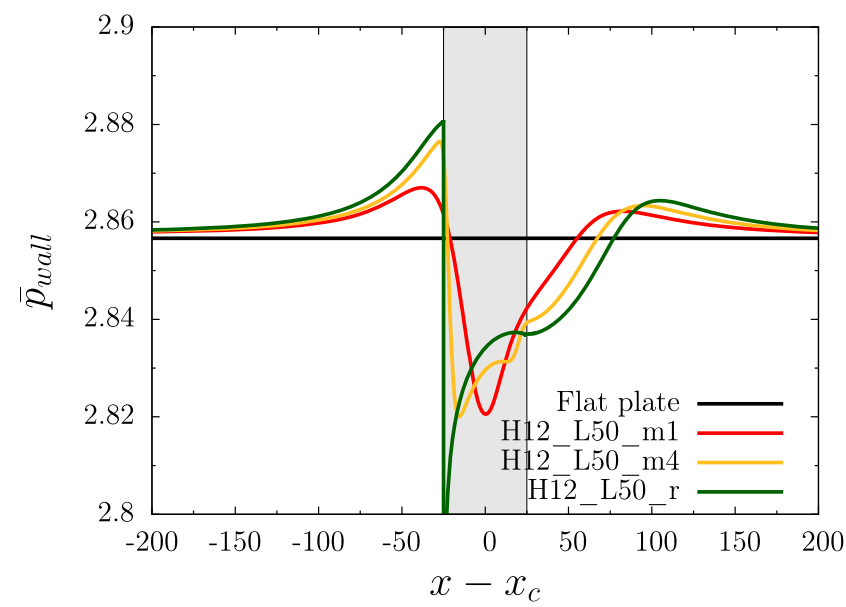

FIG. 16. Pressure distribution at the wall $\bar{p}_{\text {wall }}$ for large humps $(H=1.2)$ and length $L=50$ in cases $m=1$ (red line), $m=4$ (yellow line), and $m=r$ (green line). The flat plate case (black line) is included for comparison. The gray area represents the position of the hump.

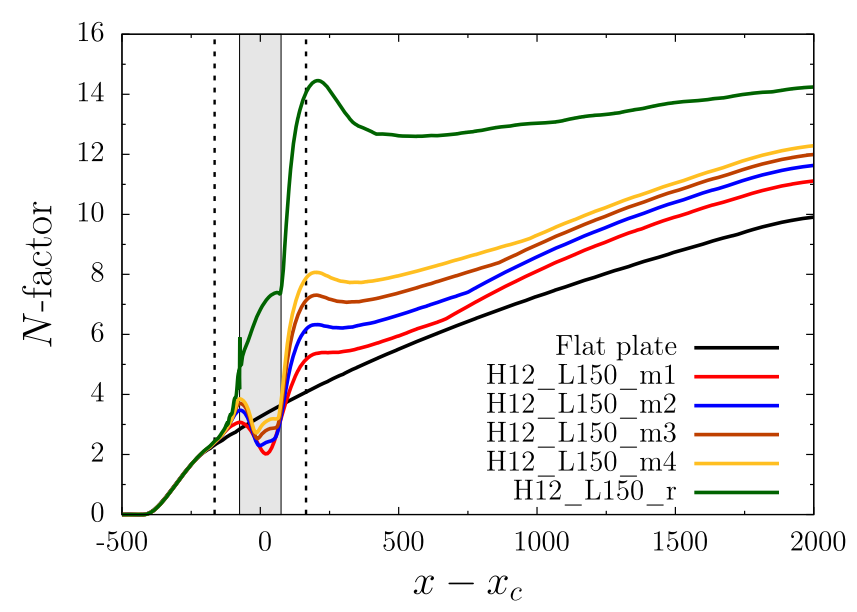

FIG. 17. $N$-factor envelope curves for large humps $(H=1.2)$ and $L=150$. Vertical dashed lines indicate the location of the interfaces between PSE and AHLNS methodologies. The gray area represents the position of the hump.

expected location where the transition to turbulence takes place. The $e^{N}$ methodology, assuming a transition $N$-factor of 9 , has been applied to all $\mathrm{N}$-factor curves previously described. The resulting expected transition locations $x_{t r}$ for the different cases are summarized in Fig. 19. Despite the shortcomings of the $e^{N}$ methodology (see Ref. 22) in predicting laminar-turbulent transition, it can still illustrate some interesting features of the different cases considered in the present work. For a flat plate, transition is expected at $\left(x_{t r}-x_{c}\right)$ $=1595.5$. When small humps $(H=0.4)$ are considered, the transition location moves slightly upstream. The change of $x_{t r}$ with respect to the flat plate case is larger when the value of the geometrical parameter $m$ is increased, although this change in transition location is relatively small $\left[\left(x_{t r}-x_{c}\right)=1515\right.$ for case H4_m1 and $\left(x_{t r}-x_{c}\right)$ $=1376.7$ for case $H 4 \_r$ ].

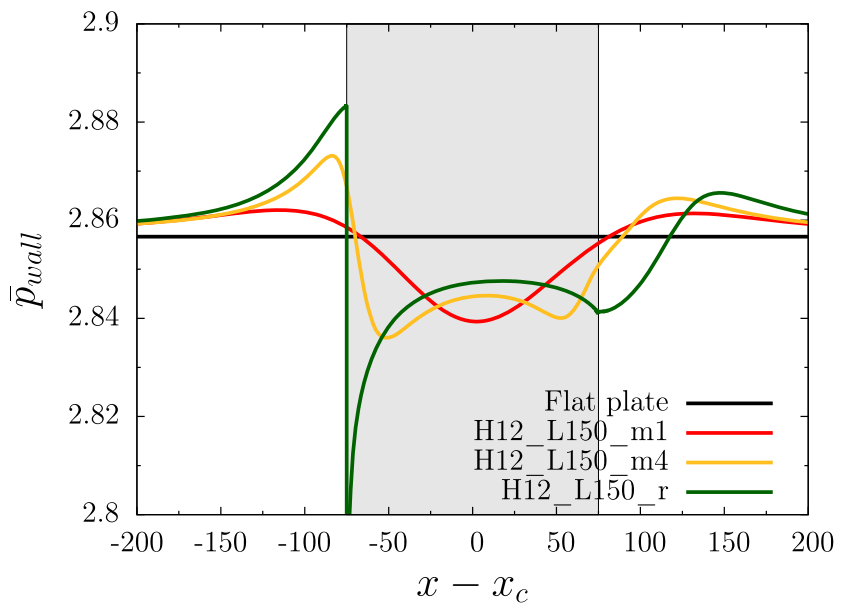

FIG. 18. Pressure distribution at the wall $\bar{p}_{\text {wall }}$ for large humps $(H=1.2)$ and length $L=150$ in cases $m=1$ (red line), $m=4$ (yellow line), and $m=r$ (green line). The flat plate case (black line) is included for comparison. The gray area represents the position of the hump. 


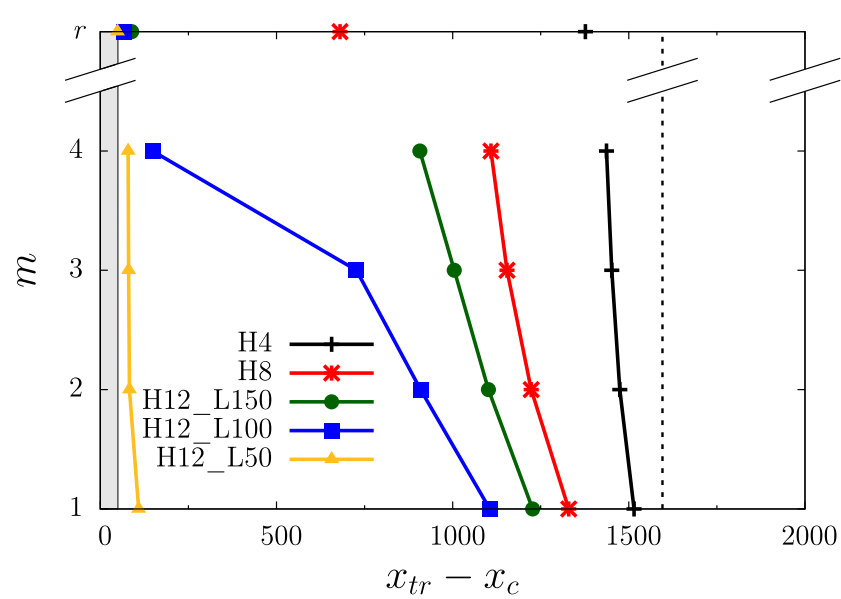

FIG. 19. Expected transition location $x_{t r}$ for all humps considered in the present work. The $e^{N}$ methodology, with a transition $N$-factor of 9 , is applied. The vertical dashed line corresponds to the flat plate case. The gray area represents the position of the hump (for $L=100$ ). Rectangular cases $(m=r)$ can be considered as limiting cases when $m \rightarrow \infty$.

For medium humps $(H=0.8)$, the transition location is more sensitive to changes in hump shape. This is especially noticeable for the rectangular case, where the expected transition location takes place at $\left(x_{t r}-x_{c}\right)=680.4$.

For large humps $(H=1.2)$, not only the shape (parameter $m$ ) was varied, but three different lengths $(L=50,100,150)$ were also considered. Transition moves upstream toward the hump by either reducing the smoothness (increasing the value of $m$ ) or reducing the length $L$. We found that, if the shape of the hump is rectangular $(m$ $=r$ ), transition to turbulence is expected to occur in the vicinity downstream of the hump. This conclusion holds for the three lengths considered in this study $(L=50,100,150)$. On the other hand, if the shape of the hump is rounded, the expected transition location $x_{t r}$ is strongly influenced by the length $L$ of the hump. Furthermore, if the length $L$ is fixed to 50 , the onset of

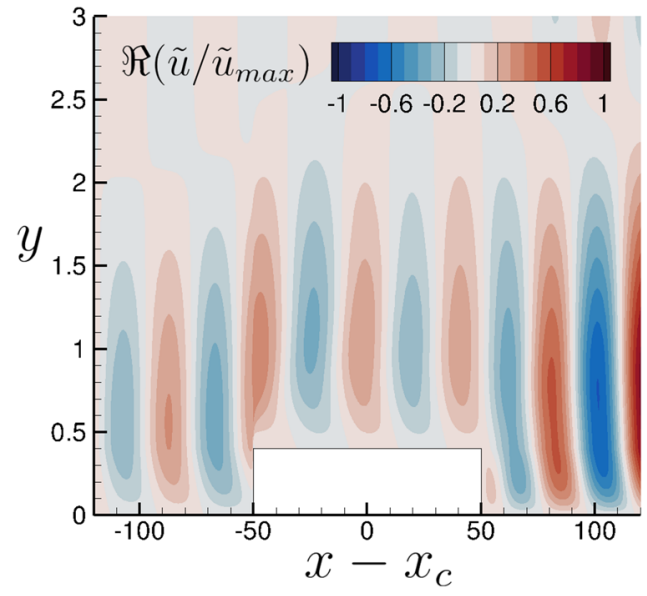

FIG. 20. Influence of the presence of a hump (case H4_r) on the real part of the streamwise velocity disturbance $\Re\left(\tilde{u} / \tilde{u}_{\max }\right)$ for an incoming TS wave of frequency $F=25$ at an arbitrary instant of time. Flow direction is from left to right. Axes are not to scale.

transition is also expected to occur in the vicinity downstream of the hump, independent of the shape of the roughness. These findings might suggest that, if transition to turbulence is intended to be significantly delayed, both length and shape of the hump should be considered simultaneously. However, we must stress that results presented here regarding the expected transition location are based on the assumption that the transition $\mathrm{N}$-factor is 9 .

\section{F. Effect of the hump on the TS wave structure}

In Secs. III C-III D, the influence of the stationary base flow pressure distribution $\bar{p}$ on the $N$-factor evolution has been described in detail for all cases considered in the present paper. In this section, we illustrate how the presence of the hump affects the spatial structure of an incoming TS wave. Figure 20 shows the isocontours

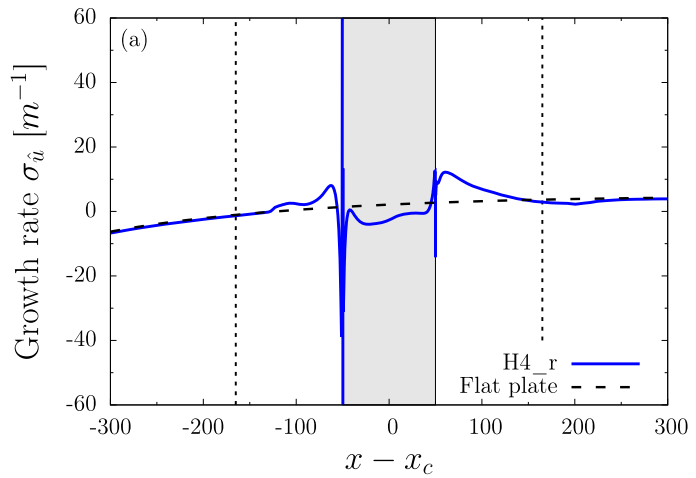

Domain $\left(x-x_{c}\right) \in[-300,300]$

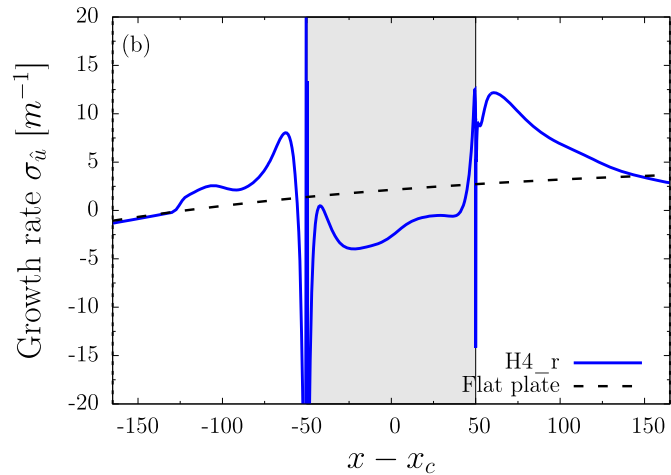

Detailed view $\left(x-x_{c}\right) \in[-165,165]$

FIG. 21. Growth rate $\sigma_{\hat{u}}$ evolution (a) for an incoming TS wave of frequency $F=25$ in the presence of a rectangular hump (case $H 4$ _ $r$ ), and a detailed view in the vicinity of the hump (b). The gray area represents the position of the hump. Vertical dashed lines indicate the location of the interfaces between PSE and AHLNS methodologies. 
of the real part of the streamwise velocity disturbance $\mathfrak{R}\left(\tilde{u} / \tilde{u}_{\max }\right)$ for a particular instant of time when an incoming TS wave of frequency $F=25$ encounters a rectangular hump (case H4_r). This particular case and frequency have been chosen due to its moderate overall variation of TS wave amplitude in the plotted domain, which facilitates a visualization by isocontours in Fig. 20. Upstream of the hump, the growth rate $\sigma_{\hat{u}}$ of the TS wave [defined in Sec. II D and depicted in Fig. 21(a) for the same case H4_r and frequency $F$ $=25$ ] and its structure are similar to the well-known development of a TS wave in a flat-plate boundary layer. Only in the vicinity of the hump [Fig. 21(b)], the structure of the TS wave changes dramatically in order to accommodate to the presence of the hump. This effect is very noticeable at the edges of the hump, especially at the upstream corner. The large variations in growth rate found at the edges of the hump when compared with the flat plate case (see Fig. 21) can be related to the base flow pressure deviations from the flat plate case (see Fig. 10): adverse pressure gradients have a destabilizing effect and, therefore, imply an increase in the growth rate of the flow instabilities. Readers can also check that variations in $\bar{p}_{\text {wall }}$ are much larger at the upstream side of the hump than at the downstream side. However, when the TS wave passes the hump, its amplitude grows much more rapidly than in the attached flow case (Fig. 9). This is due to the recirculation bubble downstream of the hump [Fig. 6(c)].

\section{G. 2D vs 3D TS waves}

Although Drazin and Reid ${ }^{26}$ stated Squire's theorem ${ }^{28}$ as "to obtain the minimum critical Reynolds number it is sufficient to consider only two-dimensional disturbances," Mack ${ }^{29}$ pointed out that this theorem only applies to incompressible self-similar boundary layers in the temporal approach, and it refers "to the minimum critical Reynolds number and not to the critical Reynolds number of a particular frequency." In the present paper, our approach is focused on the spatial development of TS disturbances along compressible boundary-layer flows where locally there is a strong streamwise

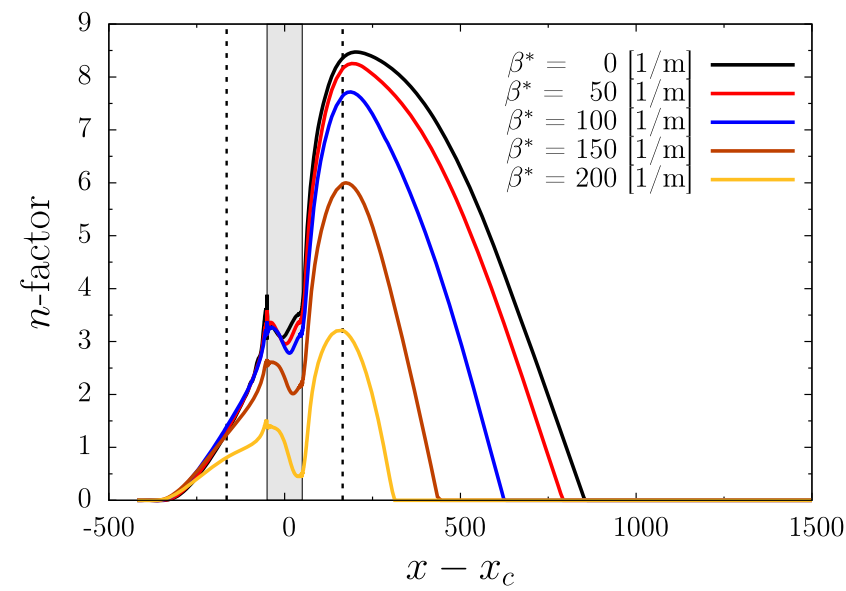

FIG. 22. $n$-factor curves for TS waves of frequency $F=48$ and several spanwise wavenumbers $\beta$. Case $H 8 \_r$ is considered. Vertical dashed lines indicate the location of the interfaces between PSE and AHLNS methodologies. The gray area represents the position of the hump. gradient caused by a surface irregularity. Therefore, it cannot be assured a priori that the most unstable disturbances are twodimensional. For this reason, we varied the spanwise wavenumber $\beta$ for a given TS wave of frequency $F=48$. The results of this investigation are shown in Fig. 22. Clearly, the maximum amplification is found for the two-dimensional wave. This assures that, for the purpose of our investigations, the two-dimensional TS disturbances are the most relevant ones in terms of $n$-factor curves.

\section{H. PSE vS AHLNS}

Some of the reasons that make the application of the PSE approach in the vicinity of the hump questionable have been previously exposed. However, it is also interesting to quantify whether these shortcomings in fact imply an incorrect computation of the $n$-factor curves. For this reason, we select the case $H 8 \_r$ and we compute the $n$-factor curve for a single TS wave of frequency $F=48$. Figure 23 shows the spatial development of the $n$-factor curve in the domain $\left(x-x_{c}\right) \in[-165,165]$ computed with AHLNS and PSE. Differences between both methods in reproducing the spatial development of the mentioned TS wave are significant, and we believe that there are two main reasons for these differences: First, the marching procedure used in PSE restricts the minimum step size (see Ref. 30) in order to avoid that the remaining ellipticity in PSE makes the numerical procedure unstable. This is a tough limitation, especially at the leading edge of the hump, where it has been shown that large flow variations occur on very short distances. Second, the PSE assume in their formulation that streamwise variations are relatively small. Again, this assumption is very questionable in the vicinity of the hump, especially in cases where sharp corners are present (see, e.g., Fig. 20).

On the other hand, we do not believe that the presence of separation bubbles with moderate reverse flow is a source of discrepancy between PSE and AHLNS. Previous studies for pressure-induced laminar separation bubbles on a flat plate (see Ref. 27) showed an excellent agreement between PSE and DNS.

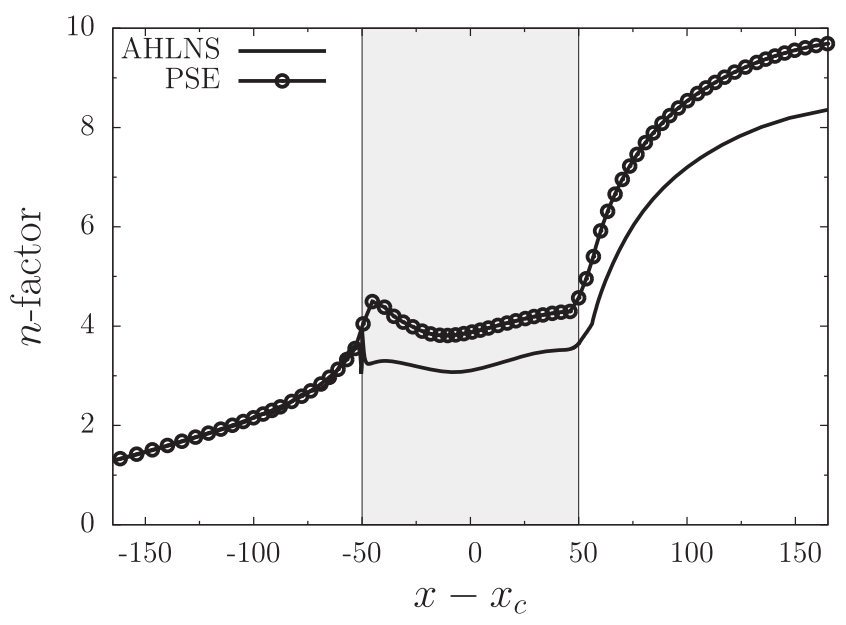

FIG. 23. $n$-factor curve for frequency $F=48$ (case $H 8 \_r$ ) computed with AHLNS (black line) and PSE (black lines with circles). Only the domain $\left(x-x_{c}\right) \in$ $[-165,165]$ is shown. The gray area represents the position of the hump. 


\section{SUMMARY AND CONCLUSIONS}

The spatial linear stability analysis of Tollmien-Schlichting waves in the presence of a large variety of two-dimensional humps on a flat plate in a compressible flow is presented here. In order to reduce the scope of all possible parameter combinations, unit Reynolds number, Mach number, and hump location have been kept fixed. The geometrical parameters, which define the hump (height, length, and shape), have been systematically varied. The shape of the hump is defined through a geometrical parameter $m$, which varies from $m=1$ (Gaussian shape) to $m \rightarrow \infty$ (rectangular shape).

The analysis shown here has been done using the recently developed AHLNS methodology, which, as has been demonstrated and justified, offers a significant reduction in the computational resources required when compared with current alternatives such as DNS solvers. Moreover, it has been explained why methodologies such as PSE or LSA have to be avoided for cases with strong streamwise gradients in the flow caused by large surface irregularities.

For each combination of height, length, and shape, the $N$-factor envelope curve has been computed. The results of the stability analysis have been related to an expected transition location via the $e^{N}$ methodology, assuming that a value of $N=9$ triggers the onset of transition to turbulence. Three different groups of humps have been considered (regarding their height): small, medium, and large. It has been found that when the height of the hump is approximately half of the boundary-layer displacement thickness $\delta^{*}$ (small humps), the transition location moves slightly upstream (compared with the flat plate case). This upstream movement of the transition onset is larger when the shape of the smooth humps tends to the rectangular shape. However, differences between rectangular and smooth humps have been found to be relatively small.

When the height is slightly smaller than $\delta^{*}$ (medium humps), the behavior of the expected transition location is similar to that of the small humps. However, differences between rectangular and smooth humps have been found to be noticeable. The rectangular hump promotes a much earlier transition location than any of the equivalent smooth humps considered.

When the height is slightly larger than $\delta^{*}$ (large humps) and its shape is rectangular, transition is expected to occur in the vicinity right downstream of the hump. This result is common for the three lengths considered in our work. On the other hand, if the large humps have a rounded shape, the transition location depends strongly on the length $L$. It can be concluded that an effective way to delay the transition onset would be to smooth the shape and to extend the length of the humps.

The effect of humps on the development of three-dimensional TS waves was also considered. It could be confirmed that, in the presence of humps, three-dimensional TS waves are still less amplified than the corresponding two-dimensional waves. Therefore, stability analysis based solely on two-dimensional waves would be enough to determine the expected transition location in subsonic two-dimensional boundary-layer flows where TS waves are the dominant instabilities triggering laminar-turbulent transition.

This systematic parametric study clearly demonstrated that the effect of surface humps on the location of laminar-turbulent transition depends on various geometrical properties of this surface irregularity. Moreover, most likely, the instability characteristics of the laminar boundary layer are of major importance as well. Therefore, attempts to assess the effect of surface irregularities on the transition location based on a simple parameter, e.g., the hump height Reynolds number, appear to have little prospect of success. Further parametric studies, which extend the scope of the present work, are required instead. The AHLNS methodology presented here is very well suited for such parametric studies as it can be applied to other types of surface irregularities, such as steps, gaps, porous walls, and suction slots, in both two-dimensional and quasi-three dimensional boundary layers.

To quantify the influence of such irregularities on the location of laminar-turbulent transition represents one of the major tasks in the design of laminar wings for future aircraft.

\section{ACKNOWLEDGMENTS}

We would like to thank the anonymous referees for their careful review of our manuscript and their insightful comments and suggestions. Part of this work was supported by the European Project NACOR (Grant Agreement No. CS2-AIR-GAM-2014-2015-01).

\section{REFERENCES}

${ }^{1}$ A. V. Boiko, A. V. Dovgal, V. V. Kozlov, and V. A. Shcherbakov, "Flow instability in the laminar boundary layer separation zone created by a small roughness element," Fluid Dyn. 25, 12-17 (1990)

${ }^{2}$ A. V. Dovgal and V. V. Kozlov, "Hydrodynamic instability and receptivity of small scale separation regions," in Laminar-Turbulent Transition, Proceedings of the IUTAM Symposium (Springer-Verlag, 1990).

${ }^{3}$ G. Danabasoglu, S. Bringen, and C. Streett, "Spatial simulation of boundary layer instability - effects of surface roughness," AIAA Paper 1993-0075, 1993.

${ }^{4}$ C. Liu and Z. Liu, "Multigrid methods and high order finite difference for flow in transition - effects of isolated and distributed roughness elements," AIAA Paper 1993-3354, 1993.

${ }^{5}$ J. A. Masad and V. Iyer, "Transition prediction and control in subsonic flow over a hump," Phys. Fluids 6, 313-327 (1994).

${ }^{6}$ A. Wörner, U. Rist, and S. Wagner, "Humps/Steps influence on stability characteristics of two-dimensional laminar boundary layer," AIAA J. 41, 192-197 (2003).

${ }^{7}$ J. Perraud, D. Arnal, and W. Kühn, "Laminar-turbulent transition prediction in the presence of surface imperfections," Int. J. Eng. Syst. Modell. Simul. 6, 162-170 (2014).

${ }^{8}$ M. Costantini, S. Risius, S. Koch, C. Fuchs, U. Gerhard, S. Hein, and C. Klein, "Experimental study of bump effects on boundary-layer transition in compressible high Reynolds number flow," Exp. Therm. Fluid Sci. 106, 234-254 (2019).

${ }^{9}$ L. Zhao, M. Dong, and Y. Yang, "Harmonic linearized Navier-Stokes equation on describing the effect of surface roughness on hypersonic boundary-layer transition," Phys. Fluids 31, 034108 (2019).

${ }^{10}$ C. Thomas, S. M. Mughal, H. Roland, R. Ashworth, and A. Martinez-Cava, "Effect of small surface deformations on the stability of Tollmien-Schlichting disturbances," AIAA J. 56, 2156-2165 (2018).

${ }^{11}$ D. Park and S. Park, "Linear and non-linear stability analysis of incompressible boundary layer over a two-dimensional hump," Comput. Fluids 73, 80-96 (2013).

${ }^{12} \mathrm{X}$. Wu and M. Dong, "A local scattering theory for the effects of isolated roughness on boundary-layer instability and transition: Transmission coefficient as an eigenvalue," J. Fluid Mech. 794, 68-108 (2016).

${ }^{13}$ M. Dong and A. Zhang, "Scattering of Tollmien-Schlichting waves as they pass over forward-/backward-facing steps," Appl. Math. Mech.-Engl. Ed. 39, 1411-1424 (2018). 
${ }^{14}$ Y. Guo, M. Malik, and C. L. Chang, "A solution adaptive approach for computation of linear waves," AIAA Paper 1997-2072, 1997.

${ }^{15}$ J. A. Franco and S. Hein, "Adaptive Harmonic Linearized Navier-Stokes equations used for boundary-layer instability analysis in the presence of large streamwise gradients," AIAA Paper 2018-1548, 2018.

${ }^{16} \mathrm{~J}$. A. Franco, S. Hein, and E. Valero, "Effect of humps and indentations on boundary-layer transition of compressible flows using the AHLNS methodology," in 7th European Conference on Computational Fluid Dynamics (ECFD 7-ECCOMAS), 2018.

${ }^{17} \mathrm{H}$. Schlichting, Boundary-layer Theory, Series in Mechanical Engineering, 6th ed. (McGraw-Hill, 1968).

${ }^{18}$ S. Hein, F. P. Bertolotti, M. Simen, A. Hanifi, and D. Henningson, "Linear nonlocal instability analysis - the linear NOLOT code," Internal Report No. DLR IB 223-94 A56, 1995.

${ }^{19}$ F. P. Bertolotti, T. Herbert, and P. R. Spalart, "Linear and nonlinear stability of the Blasius boundary layer," J. Fluid Mech. 242, 441-474 (1992).

${ }^{20}$ T. Herbert, "Parabolized stability equations," Progress in Transition Modelling, AGARD Report No. 793, 1994.

${ }^{21}$ S. Ragab and A. Nayfeh, "Stability of compressible boundary layers over a smooth backward-facing step," AIAA Paper 1990-1449, 1990.
${ }^{22}$ D. Arnal, "Boundary layer transition: Predictions based on linear theory," Progress in Transition Modelling, AGARD Report No. 793, 1994.

${ }^{23}$ C. A. Edelmann, "Influence of forward-facing steps on laminar-turbulent transition," Ph.D. dissertation (University of Stuttgart, 2014).

${ }^{24}$ v.2017.1.0, TAU-code User Guide, DLR, 2017.

${ }^{25}$ V. Theofilis, "Global linear instability," Annu. Rev. Fluid. Mech. 43, 319-352 (2011).

${ }^{26}$ P. G. Drazin and W. H. Reid, Hydrodynamic Stability, Cambridge Monographs on Mechanics and Applied Mathematics (Cambridge University Press, 1981).

${ }^{27}$ S. Hein, "Linear and nonlinear nonlocal instability analyses for two-dimensional laminar separation bubbles," in Laminar-Turbulent Transition, Proceedings of the IUTAM Symposium (Springer-Verlag, 1999).

${ }^{28} \mathrm{H}$. B. Squire, "On the stability for three-dimensional disturbances of viscous fluid flow between parallel walls," Proc. R. Soc. London, Ser. A 142, 621-628 (1933).

${ }^{29}$ L. M. Mack, "Boundary-layer linear stability," Stability and Transition of Laminar Flow, AGARD Report No. 709, 1984.

${ }^{30} \mathrm{~F}$. Li and M. Malik, "On the nature of PSE approximation," Theor. Comput. Fluid Dyn. 8, 253-273 (1996). 